Louisiana State University

LSU Digital Commons

$9-1-2018$

\title{
Characteristics and Performance of the CALorimetric Electron Telescope (CALET) Calorimeter for Gamma-Ray Observations
}

\author{
N. Cannady \\ Louisiana State University \\ Y. Asaoka \\ Waseda University \\ F. Satoh \\ Waseda University \\ M. Tanaka \\ Waseda University \\ S. Torii \\ Waseda University
}

See next page for additional authors

Follow this and additional works at: https://digitalcommons.Isu.edu/physics_astronomy_pubs

\section{Recommended Citation}

Cannady, N., Asaoka, Y., Satoh, F., Tanaka, M., Torii, S., Cherry, M., Mori, M., Adriani, O., Akaike, Y., Asano, K., Bagliesi, M., Berti, E., Bigongiari, G., Binns, W., Bonechi, S., Bongi, M., Brogi, P., Buckley, J., Castellini, G., Checchia, C., Collazuol, G., Di Felice, V., Ebisawa, K., Fuke, H., Guzik, T., Hams, T., Hareyama, M., Hasebe, N., Hibino, K., Ichimura, M., loka, K., Ishizaki, W., \& Israel, M. (2018). Characteristics and Performance of the CALorimetric Electron Telescope (CALET) Calorimeter for Gamma-Ray Observations. Astrophysical Journal, Supplement Series, 238 (1) https://doi.org/10.3847/1538-4365/aad6a3

This Article is brought to you for free and open access by the Department of Physics \& Astronomy at LSU Digital Commons. It has been accepted for inclusion in Faculty Publications by an authorized administrator of LSU Digital Commons. For more information, please contact ir@lsu.edu. 


\section{Authors}

N. Cannady, Y. Asaoka, F. Satoh, M. Tanaka, S. Torii, M. L. Cherry, M. Mori, O. Adriani, Y. Akaike, K. Asano, M. G. Bagliesi, E. Berti, G. Bigongiari, W. R. Binns, S. Bonechi, M. Bongi, P. Brogi, J. H. Buckley, G. Castellini, C. Checchia, G. Collazuol, V. Di Felice, K. Ebisawa, H. Fuke, T. G. Guzik, T. Hams, M. Hareyama, N. Hasebe, K. Hibino, M. Ichimura, K. loka, W. Ishizaki, and M. H. Israel 


\section{Characteristics and Performance of the CALorimetric Electron Telescope (CALET) Calorimeter for Gamma-Ray Observations}

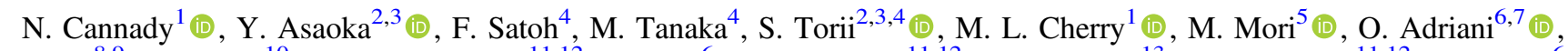
Y. Akaike ${ }^{8,9}$, K. Asano ${ }^{10}$ (1) M. G. Bagliesi ${ }^{11,12}$, E. Berti ${ }^{6}$, G. Bigongiari ${ }^{11,12}$, W. R. Binns ${ }^{13}$ (i) S. Bonechi ${ }^{11,12}$, M. Bongi ${ }^{6,7}$ (i), P. Brogi ${ }^{11,12}$, J. H. Buckley ${ }^{13}$, G. Castellini ${ }^{14}$, C. Checchia ${ }^{15,16}$, G. Collazuol ${ }^{15,16}$, V. Di Felice 17,18,19 (1), K. Ebisawa $^{20}$ (1),

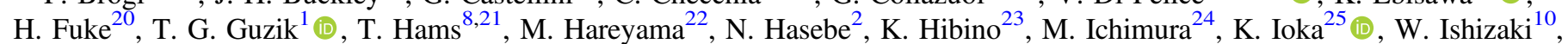

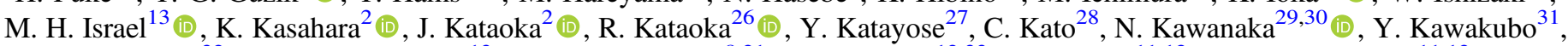
K. Kohri ${ }^{32}$, H. S. Krawczynski ${ }^{13}$ (10, J. F. Krizmanic ${ }^{8,21}$, T. Lomtadze ${ }^{12,33}$, P. Maestro ${ }^{11,12}$, P. S. Marrocchesi ${ }^{11,12}$,

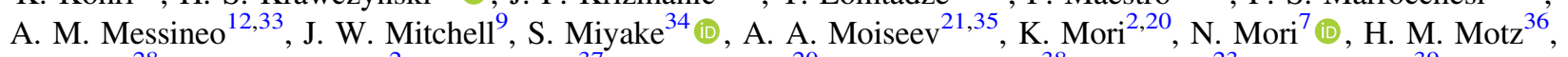
K. Munakata ${ }^{28}$ (1) , H. Murakami ${ }^{2}$, S. Nakahira ${ }^{37}$, J. Nishimura ${ }^{20}$, G. A. de Nolfo ${ }^{38}$, S. Okuno ${ }^{23}$, J. F. Ormes ${ }^{39}$, S. Ozawa ${ }^{2}$, L. Pacini ${ }^{6,714}$ (1) , F. Palma ${ }^{17,18}$, P. Papini ${ }^{7}$, A. V. Penacchioni ${ }^{11,40}$, B. F. Rauch ${ }^{13}$ (1) S. B. Ricciarini ${ }^{7}, 14$ (1) K. Sakai $^{8,21}$, T. Sakamoto ${ }^{31}$, M. Sasaki ${ }^{21,35}$, Y. Shimizu ${ }^{23}$, A. Shiomi ${ }^{41}$, R. Sparvoli ${ }^{17,18}$ (1) P. Spillantini ${ }^{6}$, F. Stolzi ${ }^{11,12}$, J. E. Suh ${ }^{11,12}$ (1), A. Sulaj ${ }^{11,12}$, I. Takahashi ${ }^{42}$, M. Takayanagi ${ }^{20}$, M. Takita ${ }^{10}$, T. Tamura $^{23}$, N. Tateyama ${ }^{23}$, T. Terasawa $^{37}$, H. Tomida $^{20}$,

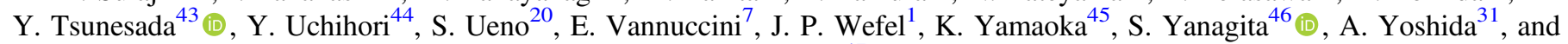
K. Yoshida ${ }^{47}$

(CALET Collaboration)

${ }^{1}$ Department of Physics and Astronomy, Louisiana State University, 202 Nicholson Hall, Baton Rouge, LA 70803, USA; ncanna1@1su.edu,

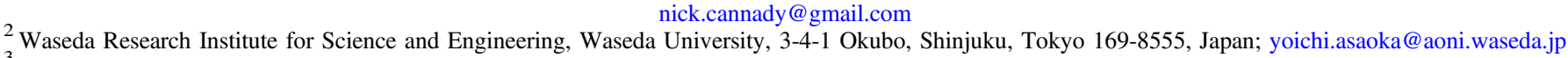

3 JEM Utilization Center, Human Spaceflight Technology Directorate, Japan Aerospace Exploration Agency, 2-1-1 Sengen, Tsukuba, Ibaraki 305-8505, Japan

${ }_{5}^{4}$ School of Advanced Science and Engineering, Waseda University, 3-4-1 Okubo, Shinjuku, Tokyo 169-8555, Japan

${ }^{5}$ Department of Physical Sciences, College of Science and Engineering, Ritsumeikan University, Shiga 525-8577, Japan

${ }^{6}$ Department of Physics, University of Florence, Via Sansone, 1, I-50019 Sesto, Fiorentino, Italy ${ }^{7}$ INFN Sezione di Firenze, Via Sansone, 1, I-50019 Sesto, Fiorentino, Italy

${ }^{8}$ Department of Physics, University of Maryland, Baltimore County, 1000 Hilltop Circle, Baltimore, MD 21250, USA

9 Astroparticle Physics Laboratory, NASA/GSFC, Greenbelt, MD 20771, USA

${ }^{10}$ Institute for Cosmic Ray Research, The University of Tokyo, 5-1-5 Kashiwa-no-Ha, Kashiwa, Chiba 277-8582, Japan

${ }^{11}$ Department of Physical Sciences, Earth and Environment, University of Siena, Via Roma 56, I-53100 Siena, Italy ${ }^{12}$ INFN Sezione di Pisa, Polo Fibonacci, Largo B. Pontecorvo, 3, I-56127 Pisa, Italy

${ }^{13}$ Department of Physics, Washington University, One Brookings Drive, St. Louis, MO 63130-4899, USA

${ }^{14}$ Institute of Applied Physics (IFAC), National Research Council (CNR), Via Madonna del Piano, 10, I-50019 Sesto, Fiorentino, Italy

${ }^{5}$ Department of Physics and Astronomy, University of Padova, Via Marzolo, 8, I-35131 Padova, Italy ${ }^{16}$ INFN Sezione di Padova, Via Marzolo, 8, I-35131 Padova, Italy

${ }^{17}$ University of Rome "Tor Vergata," Via della Ricerca Scientifica 1, I-00133 Rome, Italy

${ }^{18}$ INFN Sezione di Roma “Tor Vergata," Via della Ricerca Scientifica 1, I-00133 Rome, Italy

${ }^{19}$ Space Science Data Center-Agenzia Spaziale Italiana, via del Politecnico, s.n.c., I-00133, Roma, Italy

${ }^{20}$ Institute of Space and Astronautical Science, Japan Aerospace Exploration Agency, 3-1-1 Yoshinodai, Chuo, Sagamihara, Kanagawa 252-5210, Japan

${ }^{21}$ CRESST and Astroparticle Physics Laboratory, NASA/GSFC, Greenbelt, MD 20771, USA

${ }^{22}$ St. Marianna University School of Medicine, 2-16-1, Sugao, Miyamae-ku, Kawasaki, Kanagawa 216-8511, Japan

${ }^{23}$ Kanagawa University, 3-27-1 Rokkakubashi, Kanagawa, Yokohama, Kanagawa 221-8686, Japan

${ }^{24}$ Faculty of Science and Technology, Graduate School of Science and Technology, Hirosaki University, 3, Bunkyo, Hirosaki, Aomori 036-8561, Japan

${ }^{25}$ Yukawa Institute for Theoretical Physics, Kyoto University, Kitashirakawa Oiwakecho, Sakyo, Kyoto 606-8502, Japan

${ }^{26}$ National Institute of Polar Research, 10-3, Midori-cho, Tachikawa, Tokyo 190-8518, Japan

${ }^{27}$ Faculty of Engineering, Division of Intelligent Systems Engineering, Yokohama National University, 79-5 Tokiwadai, Hodogaya, Yokohama 240-8501, Japan

${ }_{29}^{28}$ Faculty of Science, Shinshu University, 3-1-1 Asahi, Matsumoto, Nagano 390-8621, Japan

${ }^{29}$ Hakubi Center, Kyoto University, Yoshida Honmachi, Sakyo-ku, Kyoto 606-8501, Japan

${ }^{30}$ Department of Astronomy, Graduate School of Science, Kyoto University, Kitashirakawa Oiwake-cho, Sakyo-ku, Kyoto 606-8502, Japan

${ }^{31}$ College of Science and Engineering, Department of Physics and Mathematics, Aoyama Gakuin University, 5-10-1 Fuchinobe, Chuo, Sagamihara, Kanagawa 252-5258, Japan

${ }^{32}$ Institute of Particle and Nuclear Studies, High Energy Accelerator Research Organization, 1-1 Oho, Tsukuba, Ibaraki, 3-5-0801, Japan

${ }_{33}$ University of Pisa, Polo Fibonacci, Largo B. Pontecorvo, 3, I-56127 Pisa, Italy

${ }^{34}$ Department of Industrial Engineering, National Institute of Technology, Ibaraki College, 866 Nakane, Hitachinaka, Ibaraki 312-8508, Japan

35 Department of Astronomy, University of Maryland, College Park, MD 20742, USA

${ }^{36}$ Global Center for Science and Engineering, Faculty of Science and Engineering, Waseda University, 3-4-1 Okubo, Shinjuku, Tokyo 169-8555, Japan

${ }^{38}$ RIKEN, 2-1 Hirosawa, Wako, Saitama 351-0198, Japan
Heliospheric Physics Laboratory, NASA/GSFC, Greenbelt, MD 20771, USA

${ }^{39}$ Department of Physics and Astronomy, University of Denver, Physics Building, Room 211, 2112 East Wesley Avenue, Denver, CO 80208-6900, USA

${ }^{40}$ ASI Science Data Center (ASDC), Via del Politecnico snc, I-00133 Rome, Italy

${ }^{41}$ College of Industrial Technology, Nihon University, 1-2-1 Izumi, Narashino, Chiba 275-8575, Japan

42 Kavli Institute for the Physics and Mathematics of the Universe, The University of Tokyo, 5-1-5 Kashiwanoha, Kashiwa 277-8583, Japan
${ }^{43}$ Division of Mathematics and Physics, Graduate School of Science, Osaka City University, 3-3-138 Sugimoto, Sumiyoshi, Osaka 558-8585, Japan

${ }^{44}$ National Institutes for Quantum and Radiation Science and Technology, 4-9-1 Anagawa, Inage, Chiba 263-8555, Japan

${ }^{45}$ Nagoya University, Furo, Chikusa, Nagoya 464-8601, Japan

${ }^{46}$ College of Science, Ibaraki University, 2-1-1 Bunkyo, Mito, Ibaraki 310-8512, Japan

${ }^{47}$ Department of Electronic Information Systems, Shibaura Institute of Technology, 307 Fukasaku, Minuma, Saitama 337-8570, Japan

Received 2018 March 30; revised 2018 June 26; accepted 2018 July 26; published 2018 September 5 


\begin{abstract}
The CALorimetric Electron Telescope primary detector (CALET-CAL) is a 30 radiation-length-deep hybrid calorimeter designed for the accurate measurement of high-energy cosmic rays. It is capable of triggering on and giving near complete containment of electromagnetic showers from primary electrons and gamma rays from $1 \mathrm{GeV}$ to over $10 \mathrm{TeV}$. The first 24 months of on-orbit scientific data (2015 November 01-2017 October 31) provide valuable characterization of the performance of the calorimeter based on analyses of the gamma-ray data set in general and bright point sources in particular. We describe the gamma-ray analysis, the expected performance of the calorimeter based on Monte Carlo simulations, the agreement of the flight data with the simulated results, and the outlook for long-term gamma-ray observations with the CAL.
\end{abstract}

Key words: gamma rays: general - instrumentation: detectors - methods: data analysis

\section{Introduction}

The CALorimetric Electron Telescope (CALET) is an observatory for direct, calorimetric measurement of the cosmicray electron spectrum from energies $\sim 10 \mathrm{GeV}$ well into the $\mathrm{TeV}$ region that was deployed to the International Space Station (ISS) in 2015 August (Torii et al. 2015). The primary CALET instrument is the calorimeter (CAL), which is also sensitive to photons from $\sim 1 \mathrm{GeV}$ to several $\mathrm{TeV}$, and protons and nuclei to $\sim 1 \mathrm{PeV}$. Also present on the CALET payload are the CALET Gamma-ray Burst Monitor (CGBM; Adriani et al. 2016; Yamaoka et al. 2017), which is sensitive to photons over the broad energy range $7 \mathrm{keV}-28 \mathrm{MeV}$, and the Advanced Stellar Compass (ASC; Jørgensen \& Liebe 1996), which allows for the precise determination of the CALET pointing direction (Figure 1). CALET is now docked on the Exposed Facility of the Japanese Experiment Module (JEM-EF) on the ISS. The total size is $1850 \mathrm{~mm} \times 800 \mathrm{~mm} \times 1000 \mathrm{~mm}$, with mass $612.8 \mathrm{~kg}$.

Initial results on the high-energy electron spectrum have been reported in Adriani et al. (2017). We describe here the capabilities of the CALET-CAL for GeV-energy gamma-ray observations, expanding upon previous work based on preliminary simulated data and algorithms presented in Mori et al. (2013).

\section{Instrument Description}

\subsection{The CALET Calorimeter (CAL)}

As a dedicated electron telescope, the CAL boasts a normal incidence depth of 30 radiation lengths $\left(X_{0}\right)$ and comprises three primary subdetectors (Figure 2): the CHarge Detector (CHD), the IMaging Calorimeter (IMC), and the Total AbSorption Calorimeter (TASC).

The CHD is a hodoscope made up of two orthogonal layers of plastic scintillating paddles $(32 \mathrm{~mm} \times 450 \mathrm{~mm} \times 10 \mathrm{~mm}$ each) read out by photomultiplier tubes (PMTs). It provides measurement of the absolute charge of primary particles passing through the top of the instrument.

The IMC is a sampling calorimeter (sampling fraction $\sim 12 \%$ ) with pairs of crossed $x-y$ layers ( 8 pairs $\times 2$ layers $\times$ 448 fibers) of finely segmented plastic scintillating fiber (SciFi; $1 \mathrm{~mm} \times 1 \mathrm{~mm} \times 448 \mathrm{~mm}$ each) read out by multi-anode PMTs (MAPMTs), with seven tungsten sheets interspersed between the layer pairs. The total thickness of the IMC is $\sim 3$ $X_{0}$, with the overwhelming majority of the material provided by the tungsten sheets (upper five: $0.7 \mathrm{~mm}$ each, lower two: $3.5 \mathrm{~mm}$ each). This stimulates the start and development of the particle shower, while the layers of SciFi provide high spatial resolution imaging of the cascade useful for particle identification and tracking.
The TASC is 12 crossed layers $(6$ pairs $\times 2$ layers $\times 16$ logs, each $19 \mathrm{~mm} \times 326 \mathrm{~mm} \times 20 \mathrm{~mm}$ ) of lead tungstate $\left(\mathrm{PbWO}_{4}\right.$ or PWO) logs for a total thickness of $27 X_{0}$. The top PWO layer is read out by PMTs, while the lower layers are attached to photodiode and avalanche photodiode (PD/APDs) readouts.

The depth of the calorimeter allows for nearly total containment of electromagnetic showers from primary electrons and photons with energies up to tens of $\mathrm{TeV}$. Because of this efficient collection, the reconstruction of kinetic energies requires only a small adjustment to the energy deposit sum in the TASC for electrons with energy above $\sim 10 \mathrm{GeV}$. In contrast, the calorimeter depth only corresponds to 1.3 proton interaction lengths, and a considerable fraction of the energy in showers from hadronic primaries is lost due to the escape of secondary hadrons (mainly pions). While the resultant energy resolution for hadronic primaries is worse than that for electrons and photons, the difference in shower topologies in the CAL gives a powerful rejection of the dominant proton flux.

In the discussions that follow, the CHD layers will be referred to as CHDx and CHDy, and the IMC/TASC layers will be labeled with a number from 1 to 8 and an orientation axis (e.g., IMC 1x or TASC 5y).

\subsection{Calibration}

High-quality calibration of the instrument is necessary for the accurate conversion from discrete analog-to-digital converter (ADC) units to real energy deposits. The initial calibration of the CAL was performed prelaunch with a series of laboratory tests, accelerator beam exposures, and measurement of the minimum ionizing particle (MIP) signal from penetrating cosmic-ray muons together with comparison to Monte Carlo simulations (Asaoka et al. 2017). The pedestal and MIP distributions for each channel were characterized before the launch and are updated frequently on orbit.

The detector temperature is measured by thermal sensors located at various points in the calorimeter, and a thermal model was developed preflight for the extrapolation of these measurements to every detector component. On orbit, the temperature for a given component is found to vary by a few degrees with a period of $\sim 2$ months corresponding to the change in the solar beta angle. This introduces variations of $\sim 3 \% \mathrm{rms}$ in the MIP peak position for the TASC logs. The signal from each detector is corrected based on the observed temperature dependence, reducing the variations to the order of $\sim 1 \%$ rms. The final validation of the energy response and corrections for any misalignment of the IMC fibers were performed using penetrating muon signals after the final 


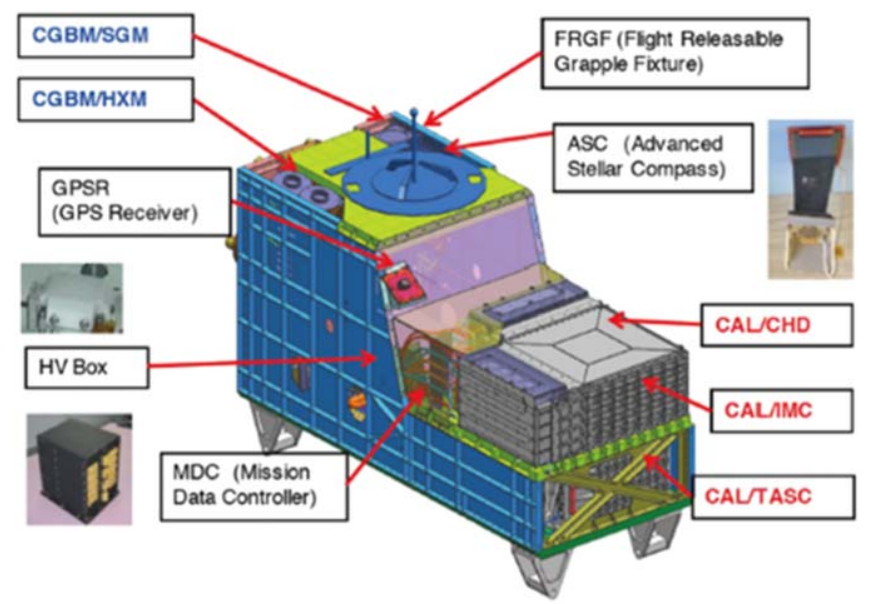

Figure 1. Locations of the CAL, CGBM, ASC, and supporting instruments on the CALET payload.

assembly of the calorimeter. In the on-orbit checkout phase, these calibrations were refined using penetrating protons and He nuclei. A detailed description is given in Asaoka et al. (2017), where an energy resolution of the summed energy deposits on the order of a few percent is achieved. The resulting error on the primary energy reconstruction for electrons (and gamma rays, given the similarity in shower shape) is estimated to be $\lesssim 3 \%$ at $10 \mathrm{GeV}$ and above, and increasing with decreasing energy to $\sim 12 \%$ at $1 \mathrm{GeV}$.

Currently, during normal scientific operations, a dedicated trigger mode for penetrating $\mathrm{He}$ is frequently enabled to update the calibrations to account for time-dependent effects. Corrections applied for temporal variations in the instrument response are briefly described in Adriani et al. (2017).

\subsection{Trigger}

Several hardware triggers are active for the calorimeter, configurable by thresholds in both CHD layers, consecutive IMC layers, and in the TASC 1x (PMT) layer (Asaoka et al. 2018). For the IMC trigger logic, the thresholds apply to the MAPMT sums, each of which represents two x-layers or two y-layers (e.g., IMC $1 x+$ IMC $2 x$ or IMC $3 y+$ IMC $4 y)$ due to the arrangement of the readouts. For gamma-ray observations up to tens of $\mathrm{GeV}$, the low-energy gamma (LE- $\gamma$ ) trigger is used when available. Active at low geomagnetic latitudes (except for the passage through South Atlantic Anomaly), the LE- $\gamma$ trigger only requires low thresholds on the bottom layers of the IMC (i.e., IMC 7x + IMC 8x, IMC 7y + IMC 8y) and the top layer of the TASC (i.e., TASC 1x), giving an effective minimum energy of $\sim 1 \mathrm{GeV}$. While there is some overlap in energy with the highenergy (HE) trigger, which has a low-energy threshold of $\sim 10 \mathrm{GeV}$, the LE- $\gamma$ trigger is assumed for the purposes of the analysis in this paper.

\subsection{Track Reconstruction}

Event tracks in the CAL are reconstructed for the LE- $\gamma$ trigger mode using the EM Track (Akaike et al. 2013), Kalman Filter Track (KF Track; Maestro et al. 2017), and CC Track (described below) algorithms. The EM Track algorithm is also used for electron analysis and is a powerful method for reconstructing electromagnetic showers. However, it has reduced efficiency for energies below $\sim 10 \mathrm{GeV}$ due to the

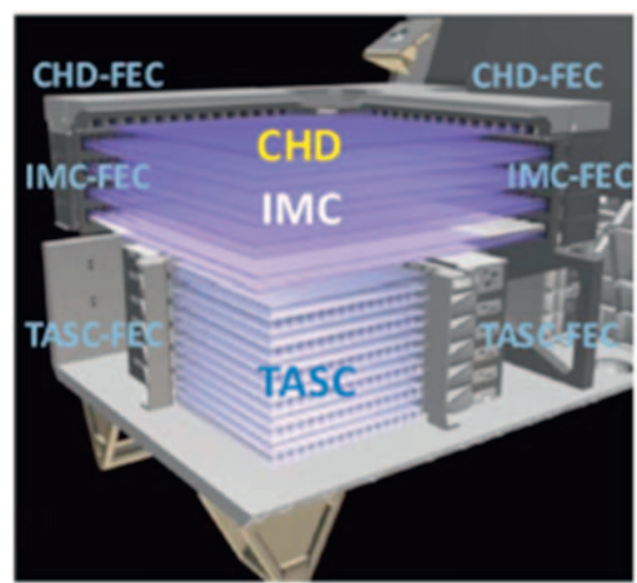

Figure 2. Schematic of the CALET-CAL.

lack of a strong signal in the bottom IMC layer for events that convert earlier in the calorimeter. Additionally, since the shower core is not as well developed for these low-energy events, the cluster of fibers in a layer with the highest energy deposit may not lie directly along the primary axis.

KF Track is designed to discriminate between a potentially large number of confusing secondary tracks. As such, it is very effective for reconstructing trajectories for protons and other nuclei. However, track quality requirements that a majority of layers be used make it inappropriate for low-energy gamma-ray events. While the trajectories fitted by this algorithm are not directly used for event reconstruction, several parameters from the procedure are useful for rejecting background proton events and are discussed further in Section 3.

CC Track is designed specifically for LE- $\gamma$ event analysis. The procedure begins with finding the five fibers with the largest energy deposits in the three bottom layers of the IMC separately for the $x$ - and $y$-projections. Clusters are formed by including the nearest neighbors to these fibers, using the center of energy for the cluster as the position in the layer to fit. Track candidates are formed by fitting the 125 ( 5 in IMC $6 \times 5$ in IMC $7 \times 5$ in IMC 8) possible combinations of these fibers. The candidate tracks are extended to the upper layers of the IMC, and additional points near the extrapolation are included in the refitting. If the reduced $\chi^{2}$ for a given track candidate increases above 2, its extension process is terminated. The energy deposits along each track candidate are summed, and the trajectory with the highest total energy deposit is selected. Using this algorithm, the number of reconstructed particle tracks in the simulated data set is increased by a factor of 2 or more compared to EM Track for photons with energies below $5 \mathrm{GeV}$.

\subsection{Event Geometries}

Event candidates are categorized in the analysis based on six acceptance conditions (with other events considered out of geometry and not used). For LE- $\gamma$ events, these geometries are referred to as $\mathrm{E}, \mathrm{ED} 3, \mathrm{~EB} 3, \mathrm{ED}, \mathrm{EB}$, and $\mathrm{A}$, ordered from least to most restrictive (Table 1). The minimum requirement for all reconstructed events is incidence on the top of the CHD and the top of the TASC, excluding a border region of $1.9 \mathrm{~cm}(1 \mathrm{PWO}$ $\log$ width). Events satisfying this condition are in acceptance condition $\mathrm{E}$ and are thus considered for analysis here. The remaining categories successively restrict the geometry and 
Table 1

Acceptance Conditions for LE- $\gamma$ Trigger Events

\begin{tabular}{lllr}
\hline \hline Geom. & & Requirements & $\left(\mathrm{cm}^{2} \mathrm{sr}\right)$ \\
\hline E & CHD top & TASC 1x top* & 373 \\
ED3 & geom. E & TASC 3y bottom & 128 \\
EB3 & geom. E & TASC 3y bottom* & 52 \\
ED & geom. E & TASC path length $>24 \mathrm{~cm}$ & 122 \\
EB & geom. E & TASC 6y bottom & 91 \\
A & geom. E & TASC 6y bottom* & 419 \\
\hline
\end{tabular}

Note. Asterisks on layers denote that a veto is used for the outer $1.9 \mathrm{~cm}(1 \mathrm{log}$ width) of the layer. The rightmost column gives the geometrical factor calculated for each condition, which is exclusively defined such that each row does not include events satisfying a condition below it.

guarantee that event trajectories have incident charges sampled by the CHD, with showers that develop within the calorimeter and without significant leakage out of the sides of the TASC. The total combined geometrical factor calculated by Monte Carlo simulation for LE- $\gamma$ analysis (Sullivan 1971) is $1184 \mathrm{~cm}^{2}$ sr. Table 1 gives the geometrical factor for each acceptance condition individually.

\subsection{Primary Energy Reconstruction}

The reconstruction of the kinetic energies of photon candidates is based on simulated photon events and is tuned separately for each acceptance condition. Because of absorption in the tungsten layers, energy deposits in the IMC are important, especially for lower energy events, where the loss in inactive detector elements is $\sim 20 \%$ at $1 \mathrm{GeV}$ and $\sim 10 \%$ at $10 \mathrm{GeV}$. In order to address this and leakage from the instrument, EPICS $^{48}$ simulations (Kasahara 1995) are used to determine appropriate scaling factors for combining the summed TASC energy deposits with the energy deposited in the scintillating plastic layers of the IMC (Akaike et al. 2015).

\section{Event Classification and Effective Area}

Given the procedures for the reconstruction of the primary trajectory and kinetic energy along with the definitions for the acceptance conditions described previously, the selection of photon events will now be described in detail. In this section, the specifics of the various cuts used in the gamma-ray selection are discussed, and the resulting effective area is evaluated using EPICS simulated data. We generated photons isotropically in EPICS on a partial sphere covering the CAL and extending down to incidence angles of $90^{\circ}$. The thrown energies span the range from $100 \mathrm{MeV}$ up to $1 \mathrm{TeV}$ following an $E^{-1}$ distribution to equally populate bins equally spaced in logarithm. A total of $3.2 \times 10^{8}$ events were generated per decade of energy.

\subsection{Gamma-Ray Selection}

Initial preselection conditions are necessary to isolate a sample of events that can be reconstructed with sufficient accuracy for this analysis and to guarantee the validity of the efficiencies derived from simulated data. Note that the analyses for the EM Track and CC Track algorithms are performed separately but that the conditions on the events are the same at

\footnotetext{
48 http://cosmos.n.kanagawa-u.ac.jp/EPICSHome/
}

the selection level regardless of the tracking algorithm. These cuts have been optimized for the LE- $\gamma$ event analysis. The charge cut has been modified from previous CALET presentations (e.g., Cannady et al. 2017; Mori et al. 2017), and the albedo, KF Track, and field-of-view (FOV) cuts are new additions.

Offline trigger. In the hardware trigger for flight data, the logical discriminators are configured in terms of the ADC units for the thresholds. When translated into deposited energies, temporal variations are present due to the absorption of light in the scintillators and the temperature and position dependence of the response characteristic of each channel among other effects. To mitigate this variability inherent in the flight data sample, an offline trigger is applied to both flight and simulated events with a threshold on the summed energy deposits in the LE- $\gamma$ trigger layers (Section 2.3). Specifically, the requirements for the LE- $\gamma$ offline trigger are 7 MIPs in IMC $7 x+$ IMC $8 x, 7$ MIPs in IMC $7 y+$ IMC $8 y$, and 10 MIPs in TASC $1 x$. As shown in Figure 3, the sensitivity drops to $\sim 50 \%$ at $1 \mathrm{GeV}$, reaching zero at $\sim 500 \mathrm{MeV}$. For the analysis presented in this paper, only those events reconstructed with kinetic energy $1 \mathrm{GeV}$ or higher are considered.

Tracking. First, basic requirements are imposed on the trajectories of the events. For the purpose of evaluating the effective area, the simulated event sample is trimmed to only include those with Monte Carlo true trajectories satisfying the geometry E conditions (Table 1). The more general subsequent filter applied to both simulated and flight data requires a reconstructed track which satisfies the geometry E conditions.

The number of IMC layers used in the track reconstruction is taken to be a proxy for the height in the instrument where the initial pair conversion interaction occurs. The numbers corresponding to the $x$ - and $y$-projections of the track are referred to as $N_{p_{x}}$ and $N_{p_{y}}$, respectively. To guarantee reliable tracking, $N_{p_{x}}$ and $N_{p_{y}}$ are both required to be $\geqslant 3$, and the condition

$$
\left|N_{p_{x}}-N_{p_{y}}\right| \leqslant 1
$$

is imposed to reject inconsistent estimates of the pair conversion layer, as this suggests poor reconstruction for one or both axes. Furthermore, events that are reconstructed with $N_{p_{x}}=8$ or $N_{p_{y}}=8$ are found to be generally misreconstructed for gamma-ray analysis and are much more common for hadronic primaries. As a result, events using all eight IMC layers in the tracking are also removed.

The final requirements on the quality of the reconstructed track are in the consistency between the direction and the energy deposits in the bottom of the IMC and the top of the TASC. Energy-dependent constant-efficiency cuts were tuned using EPICS gamma-ray data separately for each of these conditions. The total energy in IMC 8 is required to surpass a threshold based on $98 \%$ acceptance of otherwise well-tracked simulated events in the same geometrical acceptance condition. The distance between the projected intersection of the track with TASC $1 \mathrm{x}$ and the center of energy in that layer is calculated and must not exceed a threshold set at $98 \%$ of events passing the previous cut that have an $N_{p_{x}}$ or $N_{p_{y}}$ of 3 , since these represent the least accurate events that are acceptable for analysis.

Shower shape/hadronic rejection. Low-energy gamma-ray events with a small shower and zero charge measurement can 

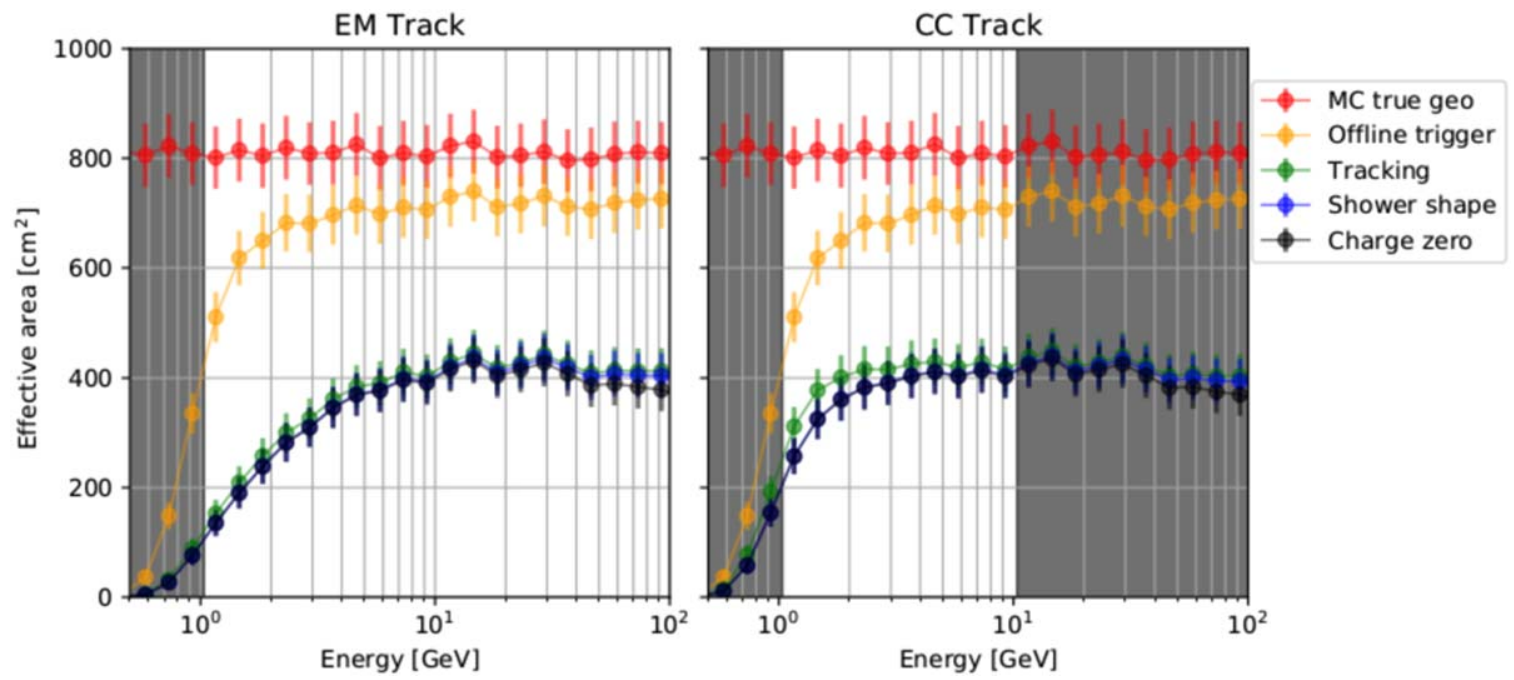

Figure 3. Effect of selection cuts in zenith-pointing effective area. Gray shaded regions demonstrate the limits of applicability for each tracking algorithm due to background contamination and poor agreement between flight data and simulation.

be mimicked by albedo (i.e., upward moving) secondary charged pions from hadronic interactions in the calorimeter or the support structure for the instrument. A signature for upward moving, stopping particles would be an increase in the energy deposit per unit length in the detector upward along the reconstructed track. To veto these events, gamma-ray candidates are required to deposit more energy in the bottom layer of the IMC than in the layer of pair conversion.

Further rejection of events with showers not consistent with a well-tracked pure electromagnetic cascade is provided by a cut on the IMC concentration. This quantity uses the lateral spread of the energy deposit distribution in the lower layers of the IMC. For IMC 8x and IMC 8y separately, it is required that the fraction of energy deposited within \pm 1 Molière radius for the tungsten sheets of the reconstructed track (corresponding to \pm 9 SciFi fibers) be at least $40 \%$ of the energy deposited in each layer in total.

Several parameters from KF Track have physical meaning for events in the CAL and are used in the rejection of the hadronic background for the gamma-ray analysis. For every event in the EM Track and CC Track samples, the KF Track is attempted. A limit is placed on the number of clusters found for tracking in the IMC at 400 , and a veto is placed on any event with a well-fitted KF Track result, which differs from the EM Track or CC Track (depending on which is being considered) reconstruction by more than $6^{\circ}$. The final requirement on the shower shape of the events utilizes the $K$ parameter. $K$ is defined as

$$
K=\log _{10}\left(F_{E}\right)+R_{E} / 2 \mathrm{~cm},
$$

where $R_{E}$ is the second moment of the lateral energy deposit distribution in the top layer of the TASC, and $F_{E}$ is the fractional energy deposit in the bottom TASC layer with respect to the total energy deposit sum in the TASC. This estimator is one of two methods used for the e/p separation in the derivation of the electron flux (Adriani et al. 2017) and is designed to exploit the larger spread and slower development of proton showers due to the penetrating nature of secondary hadrons.

Charge zero. In order to select events consistent with a primary charge of zero, cuts are made on the energy deposits in the CHD and upper IMC layers. These requirements are designed to veto events with consistent charge $>1$ MIPs in at least one of three filters. The three filters include (1) CHD max, the sum of the maximum energy deposit in a paddle and the larger of its neighbors in each axis, (2) CHD hit, the sum of energy deposits in the three paddles nearest the reconstructed track for each axis, and (3) IMC hit, the sum of energy deposits in the nine fibers nearest the track for each axis. The cuts were initially studied with EPICS simulated electron, proton, and photon data sets. The thresholds were validated by comparing the analogous distributions in the flight data sample. The specific requirements, their efficiencies, and a representative distribution for one of the filters are shown in Figure 4.

Field-of-view limit. Several fixed structures on the ISS are visible in the FOV of the CAL when considering geometry $\mathrm{E}$ events. Cosmic-ray interactions in these structures create secondary photons that are detected by the CAL and create a constant photon background for the gamma-ray analysis. A map of photon candidates in the CAL-frame coordinates and the locations of ISS structures were used to create a mask for the reconstructed trajectories, effectively removing these structures. This mask is included in the calculation of the effective area and is thus accounted for in the exposures.

\subsection{Effective Area}

The resulting effective area of the CAL to gamma rays is derived using simulated data sets. To model the response, the FOV of the CAL is divided into equal solid angle pixels using the HEALPix scheme (Gorski et al. 2005). The simulated events are thrown with an isotropic distribution over a hemisphere covering the top half of the calorimeter. For energy bin $i$ and sky pixel $j$, the effective area is calculated as

$$
S_{\mathrm{eff}}^{i j}=\frac{(S \Omega)_{\mathrm{inc}}}{(\Delta \Omega)} \frac{N_{\mathrm{acc}}^{i j}}{N_{\mathrm{inc}}^{i}}
$$

where $N_{\mathrm{acc}}^{i j}$ is the number of photon candidates passing the selection, $N_{\mathrm{inc}}^{i}$ is the number of photon candidates thrown, $(S \Omega)_{\text {inc }}$ is the geometrical factor of the throw surface, and $(\Delta \Omega)$ is the size of one sky pixel. 

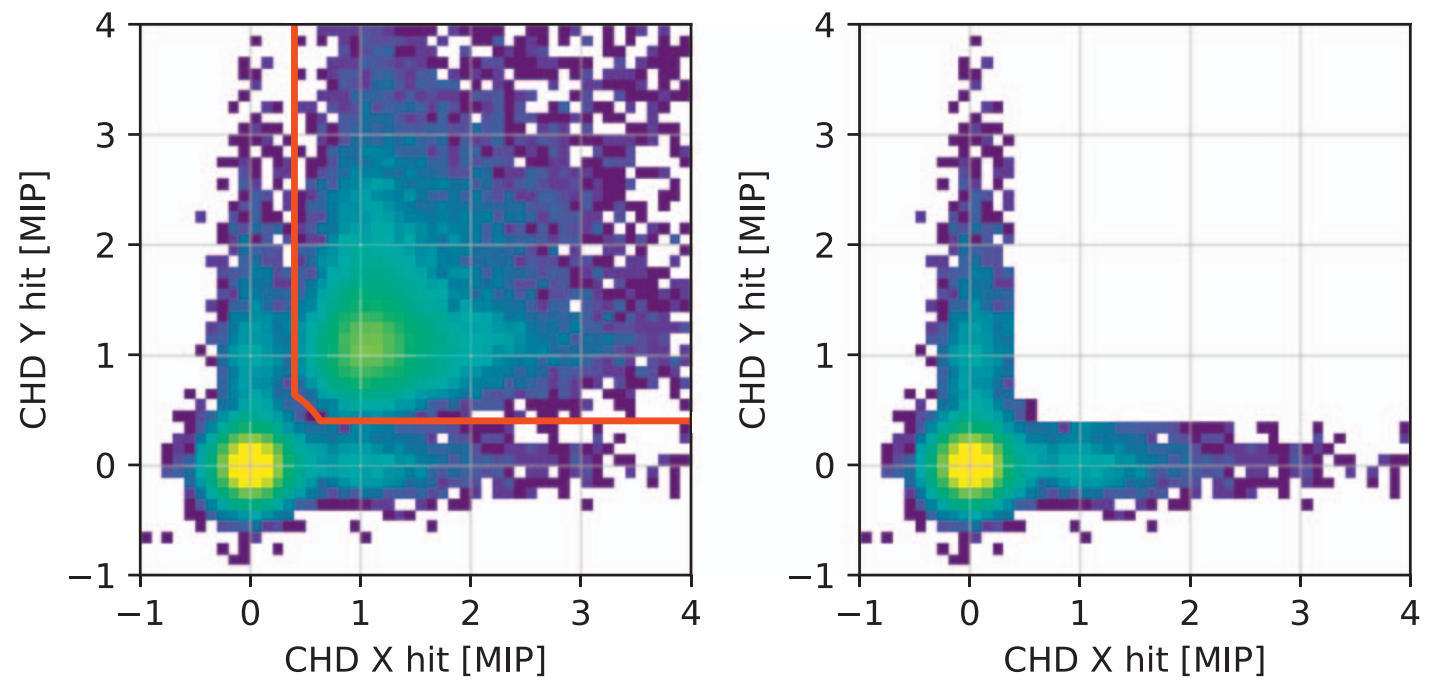

\begin{tabular}{|c|c|c|c|c|c|c|c|}
\hline \multirow[t]{2}{*}{ Cut } & \multirow[t]{2}{*}{ Requirement } & \multicolumn{2}{|c|}{$\mathrm{e}^{-}$} & \multicolumn{2}{|c|}{$\mathrm{p}$} & \multicolumn{2}{|c|}{$\gamma$} \\
\hline & & $n_{\text {pass }}$ & & $n_{\text {pass }}$ & & $n_{\text {pass }}$ & \\
\hline Preselection & & 423 & $100 \%$ & 870 & $100 \%$ & 1319418 & $100 \%$ \\
\hline CHD hit & $X<0.4$ or $Y<0.4$ or $\sqrt{X^{2}+Y^{2}}<0.75$ & 12 & $2.84 \%$ & 216 & $24.8 \%$ & 1317410 & $99.9 \%$ \\
\hline CHD max & $X<1$ or $Y<1$ or $\sqrt{X^{2}+Y^{2}}<2$ & 7 & $1.65 \%$ & 141 & $16.2 \%$ & 1314070 & $99.6 \%$ \\
\hline IMC hit & $X<0.1$ or $Y<0.1$ & 7 & $1.65 \%$ & 139 & $16.0 \%$ & 1313993 & $99.6 \%$ \\
\hline
\end{tabular}

Figure 4. Charge selection criteria using the CHD and IMC. Top: distribution of signals in the CHDy hit strip vs. CHDx hit strip (left and right panels show before and after this cut, respectively) for CC Track events $(1 \leqslant E[\mathrm{GeV}] \leqslant 10)$ in flight data. The red line indicates the cut boundary, with events in the upper-right region rejected by the filter. Bottom: requirements of the main charge zero selection filters and the simulated effect (number and fraction remaining in the sample) on electrons, protons, and gamma-ray samples reconstructed with the CC Track passing the offline trigger, tracking requirements, and shower shape.

The effect of successive cuts on the effective area is shown for events near normal incidence for both EM Track and CC Track in Figure 3. Figure 5 shows the variation of the final selection with zenith angle. Note that the large fluctuations in the bins with a high incidence angle are due to the FOV restrictions, which introduce non-uniformity in the response. The maximum of the effective area is achieved at approximately $10 \mathrm{GeV}$, with the sensitivity at higher energies decreasing due to contamination from backscattered particles in the CHD signal. Variations of the types of cuts described above are in development for gamma-ray analysis using the HE trigger and the EM Track reconstruction for events above $\sim 30 \mathrm{GeV}$ to mitigate this loss of sensitivity by including charge measurement with high spatial resolution in the upper layers of the IMC.

\section{Point-spread Function and Angular Resolution}

The procedure for determining the angular resolution and point-spread function (PSF) of the CAL for gamma-ray observations follows the treatment in Ackermann et al. (2012) for Fermi-LAT.

\subsection{Derivation from Simulated Data}

Starting from the simulated data set described previously in Section 3, the selection conditions are applied to obtain a representative sample of photon candidates. For each event, the angular error $\alpha$ in the track reconstruction is calculated using the Monte Carlo true direction and that obtained from the fit.
In general, the response of the calorimeter is a function of many parameters, due to the intrinsic resolution of the detector and systematic effects of the analysis. The most significant considerations are the kinetic energy of the particle and $N_{p}$, taken for each event to be the smaller of $N_{p_{x}}$ and $N_{p_{y}}$ (as defined in Section 3.1, tracking). The angular resolution, $C_{68}$, is determined in each energy and $N_{p}$ bin as the radius of the angular error for which $68 \%$ of events are reconstructed with $\alpha<C_{68}$. This quantity is well represented for the CAL by the functional form

$$
S_{p}\left(E, N_{p}\right)=\sqrt{\left(c_{0} E^{-\beta}\right)^{2}+c_{1}^{2}} \times\left(1+E^{\delta}\right),
$$

where the functional parameters $\left(c_{0}, c_{1}, \delta, \beta\right)$ are themselves dependent on $N_{p}$, and $E$ is in units of GeV. This form is similar to that used for the scaling function described in Ackermann et al. (2012). It differs by the inclusion of the $N_{p}$ dependence and the factor $\left(1+E^{\delta}\right)$, which was added to account for the increasingly detrimental effect of backscattered particles on the effective reconstruction of tracks in the CAL with increasing primary photon energy. Optimal parameters are found by a chisquared fitting of the $C_{68}$ calculated from the simulated distributions. Figure 6 shows these values for the angular resolution and the agreement with the fitted $S_{p}$.

The PSF, $P$, is constructed to represent the probability density of reconstructing an event at a given $\alpha$. It is therefore expected to be normalized in solid angle over the possible 

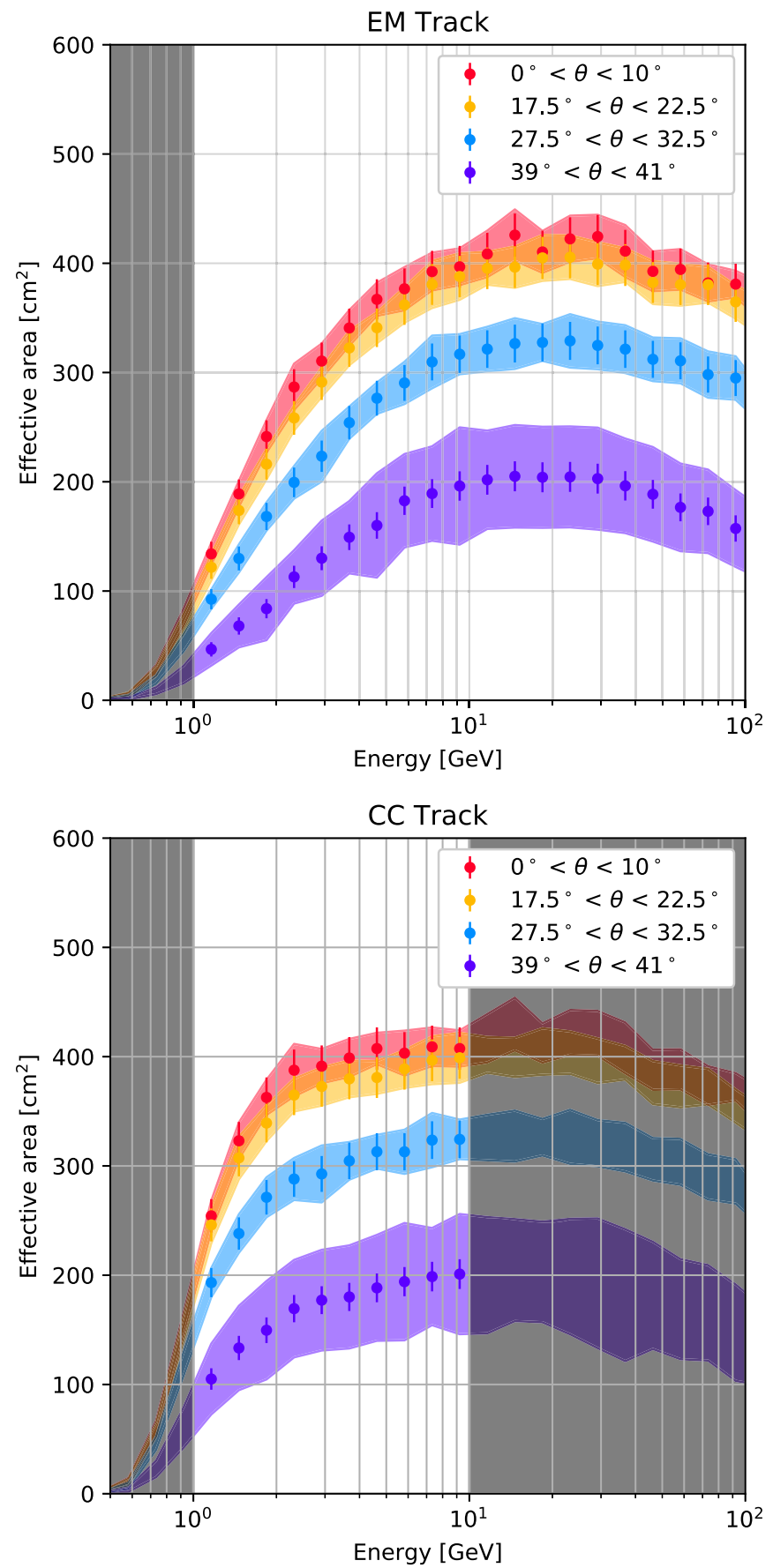

Figure 5. Effective area for events at varying zenith angle bins for EM Track and CC Track. Filled curves show the $1 \sigma$ variation in each zenith bin, and the error bars are the statistical error in the bin. Bins were chosen for comparable photon numbers to reduce the statistical error arising from small widths, which are used to reduce the effect of the changing sensitivity within each bin. Note the increased sensitivity of the CC Track algorithm at low energies.

angular errors,

$$
\int_{0}^{\pi} P(\alpha) 2 \pi \sin (\alpha) d \alpha=1
$$

If the PSF decreases sufficiently rapidly that the contribution to the integral from angles where the small-angle approximation, $\sin \alpha \sim \alpha$, fails is $\lesssim 1 \%$, the normalization condition can be

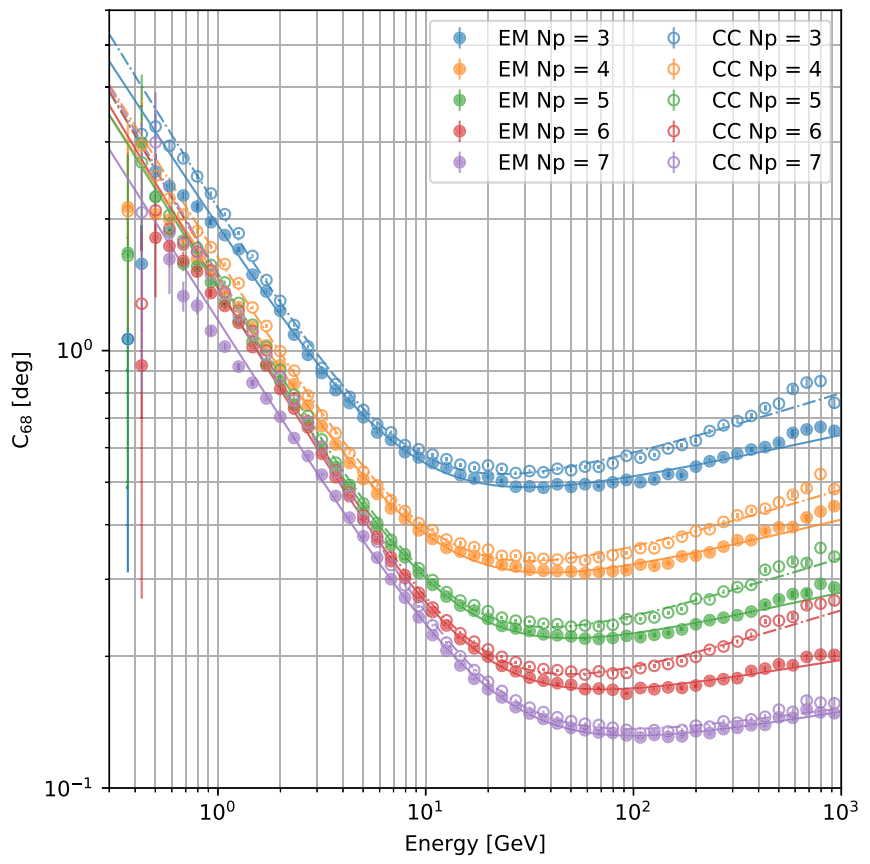

Figure 6. $C_{68}$ as a function of energy and $N_{p}$ for both tracking algorithms. Smooth curves show the fits for $S_{p}$.

simplified, i.e.,

$$
\int_{0}^{\pi} P(\alpha) 2 \pi \alpha d \alpha=1
$$

We find that the function used to describe the PSF of FermiLAT (a King function; King 1962) is also appropriate for the distribution of angular errors in the CAL. The functional form used in Ackermann et al. (2012),

$$
K(\alpha, \sigma, \gamma)=\frac{1}{2 \pi \sigma^{2}}\left(1-\frac{1}{\gamma}\right)\left[1+\frac{1}{2 \gamma} \frac{\alpha^{2}}{\sigma^{2}}\right]^{-\gamma},
$$

satisfies the normalization condition

$$
\int_{0}^{\infty} K(\alpha) 2 \pi \alpha d \alpha=1
$$

by choice of its parameters. If the PSF decays sufficiently quickly, the error introduced by integrating beyond the upper limit of $\pi$ is negligible. A pair of King functions to describe a core and a tail contribution to the PSF are necessary to best match the CAL response.

We scale the angular errors $\alpha$ to units of the angular resolution according to the functions fitted to the angular resolution above,

$$
x=\frac{\alpha}{S_{p}\left(E, N_{p}\right)},
$$

where $x$ represents this scaled angular error. Constructing the PSF as a function of $x$ rather than $\alpha$, we find that the application of the scaling functions yields a response independent of photon energy and $N_{p}$. For the global PSF, we obtain the distributions shown in Figure 7. The core and tail contributions and the composite fit are shown for both EM Track and CC Track by the red, green, and blue curves, respectively. These correspond to $68 \%, 95 \%$, and $99 \%$ containment of events at 

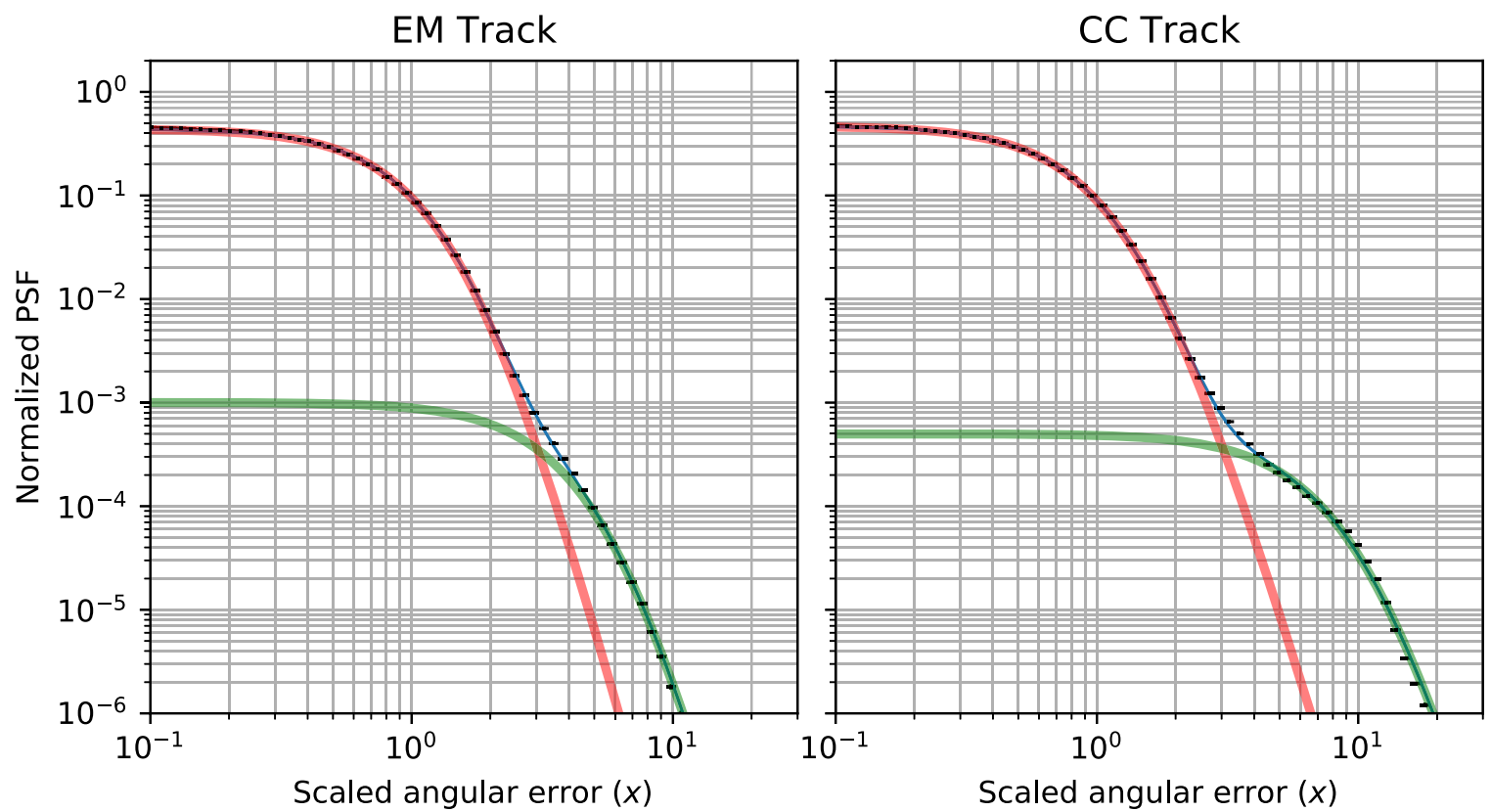

Figure 7. Composite PSF for EM and CC tracks. In each plot, the core contribution and tail contribution are represented by the red and green curves, respectively.

$x$-values of 1 (1), 1.9 (2.0), and $2.6(3.5)$, respectively, for EM Track (CC Track) events.

We note that the normalization (Equation (2)) of the King functions remains valid for the scaled quantity $x$ if the assumptions hold separately for all bins considered. That is, the normalization conditions are identical in $\alpha$ and $x$ for a given bin under appropriate scaling of the width, $\sigma_{\alpha}=S_{p}\left(E, N_{p}\right) \sigma_{x}$. For the energy range above $1 \mathrm{GeV}$, it is found that the error implicit in the small-angle approximation is no more than $\sim 1 \%$ out to $x$ of 10 or more. Furthermore, the error introduced by extending the limits of integration is validated numerically and is found to be negligible $\left(\sim 10^{-4}\right)$.

\subsection{Isolation of Photon Candidates from Bright Point Sources}

To evaluate the consistency of these results with the observed performance of the instrument on orbit, we isolate signals from point sources that are in exposed regions of the sky and are bright enough to be significant over the background in the CAL analysis. The most prominent sources are the Crab, Vela, and Geminga pulsars and the active galactic nucleus (AGN) CTA 102 seen during a bright flare in late 2016 to early 2017.

In order to determine the arrival directions for photon candidates, a transformation from the CAL reference frame to a celestial system must be defined. Orientation information for the CAL is primarily determined using the ASC, which determines its orientation by correlating images of the sky and star maps. Since this information is not always available or reliable due to the position of the Sun or high residuals in the correlation, the ASC quaternions ${ }^{49}$ must be interpolated over regions with missing information (Asaoka et al. 2018). For short time gaps, spherical linear interpolation can be used. For longer gap periods, the orientation quaternion of the ISS must be used. Since the orientation of the ISS is not exactly aligned

\footnotetext{
${ }^{49}$ Quaternions are four-dimensional objects similar to vectors with unique rules for multiplication. They allow for a straightforward calculation of rotations between orientations.
}

Table 2

Error in the Mean Position of Candidates Associated with Different Point Sources Before and After Application of the Correction Quaternion

\begin{tabular}{lcc}
\hline \hline Source & Error, pre [deg] & Error, post [deg] \\
\hline Crab & 0.11 & 0.049 \\
CTA 102 & 0.12 & 0.048 \\
Geminga & 0.047 & 0.018 \\
Vela & 0.19 & 0.088 \\
\hline
\end{tabular}

with that of the ASC, an additional correction quaternion is tuned when both ASC and ISS data are available and applied for the gap periods. Using flight data with ASC data artificially removed, this correction provides stable consistency between corrected ISS quaternions and the ASC quaternions with error $<0^{\circ} .2$.

\subsection{Absolute Pointing Accuracy}

To determine the absolute accuracy in our determination of pointing direction, we take the Fermi-LAT 3FGL (Acero et al. 2015) to be a self-consistent catalog and calculate the error between the catalog position and the most likely position based on CAL flight data. The difference between the CAL positions and the Fermi-LAT positions for the Crab, Vela, Geminga, and CTA 102 are listed in the middle column of Table 2. In order to mitigate any systematic shifts in the calculated arrival direction imposed by an overall rotation between the coordinate systems used by the ASC and FermiLAT, a correction quaternion is introduced. Both the most likely positions (Figure 8) and the components for the correction quaternion are determined by a log-likelihood minimization of the PSF with the MINUIT software. MIGRAD and MINOS errors for the source localization are mutually consistent and range from $\sim 0^{\circ} .014$ for Geminga to $\sim 0^{\circ} .038$ for Vela.

After application of this fine adjustment to the arrival directions of the candidates, we find a decrease in the difference between the 3FGL position and the observed position in the CAL (right-hand 

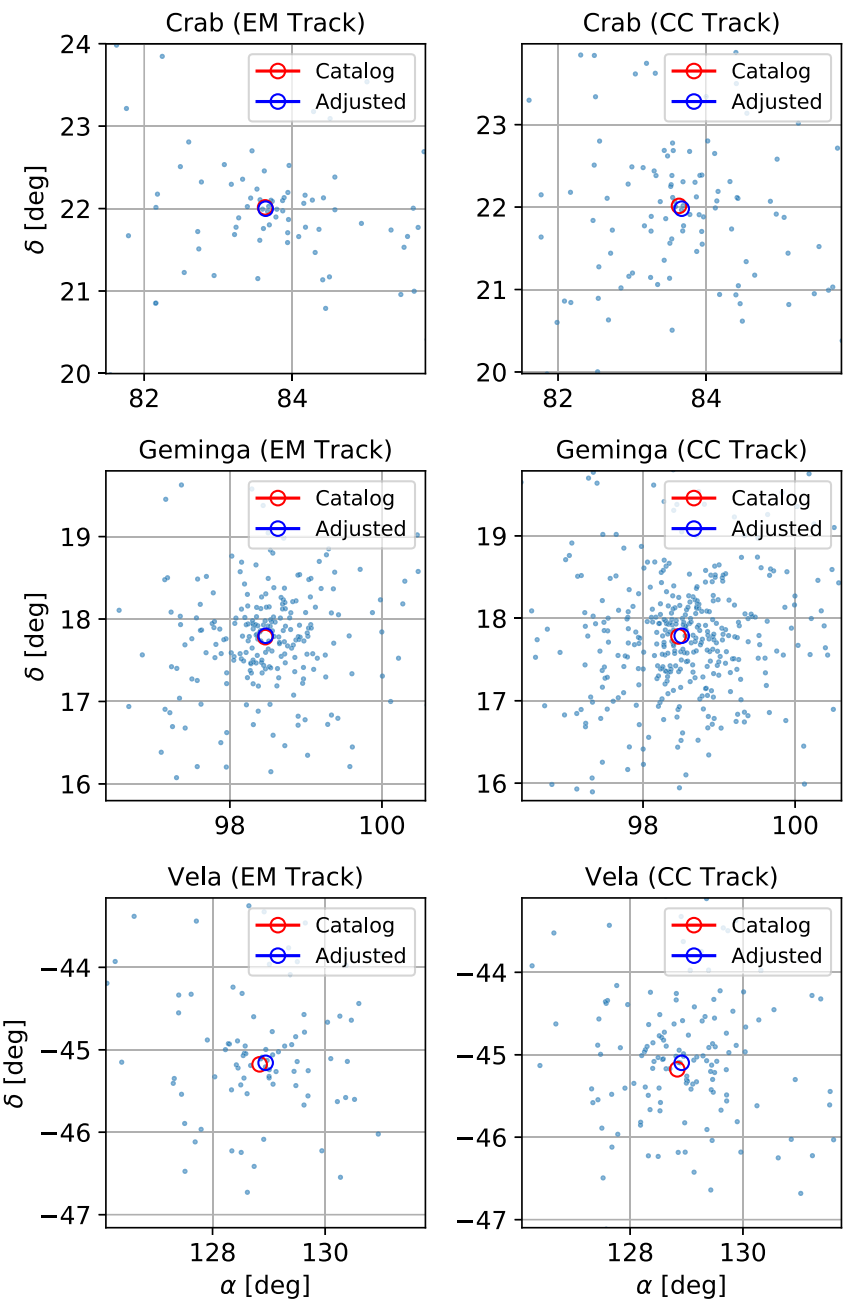

Figure 8. Catalog and most likely positions based on the candidate events for the three pulsars studied.

column, Table 2). The remaining discrepancies are random in direction and cannot be further improved by the application of a successive rotation. They represent the current limit of CALET to determine these positions based on the available photon statistics and any systematic errors present in the transformation. These errors are well below the expected CALET position resolution of 0 . 1 for the gamma-ray analysis and demonstrate the long-term stability of the ISS quaternion correction and the capability for direction determination in the CAL.

\subsection{Validation of Simulated Results with Flight Data}

Using the known positions of the sources, we generate distributions from the flight data analogous to the simulated PSF. The angular distance for each event from its associated source is determined and scaled using the scaling functions $S_{p}$ (Equation (1)). As shown in Figure 9 for the case of Geminga, there exists a plateau due to the background of photons and charged particles. This plateau becomes significant at $x \gtrsim 2$, which corresponds to the $95 \%$ photon containment radius. To account for this effect, we add a constant background tuned using the level of the plateau to each of the PSF fits. Consistency between the distributions after this correction demonstrates that the flight data are well represented by the simulated results. The source of the background is discussed in detail in the following section.

\section{Gamma-Ray Observations}

\subsection{Gamma-Ray Background from ISS Structure}

As discussed briefly in Section 3, cosmic-ray interactions with ISS structures create a secondary photon background for gamma-ray analyses using the CAL. While a mask is used to remove permanent obstructions from the FOV and is applied in the event selection, the observed signal from moving structures such as the radiator, solar panels, supply ships, and robotic arms is the primary source of background in long-term observations (Figure 10).

The obstructions can be categorized as quasi-fixed or transient based on their predictable (i.e., regular appearance of the solar panels in the FOV due to rotation) or unpredictable (i.e., passage of a robotic arm into the FOV for an extended period of time) behavior. Time-dependent positioning of some structures such as the JEM Remote Manipulator System is currently determined. While times of activity can be determined for unpredictable obstructions such as by the Space Station Remote Manipulator System (SSRMS), evaluation of the amount of blocking occurring for some time frames is still underway. A robust solution for removing the background events in the direction of these structures is under development. At this stage of analysis, however, sections of the FOV are vetoed at the event selection stage based on monthly maps of regions significantly affected.

\subsection{Comparison with Expectation from Fermi Observations}

To evaluate the current understanding of the background in the LE- $\gamma$ analysis, we compare the CAL distribution of candidate events on the sky with the expectation from FermiLAT observations. Fermi-LAT Pass 8 data were retrieved from the public archive ${ }^{50}$ for the dates 2008 August 04 through 2017 March 12 and used to calculate an observed flux map for the energy range 1-100 GeV. As discussed in Asaoka et al. (2017), an absolute energy scale correction of $3.5 \%$ is introduced in the electron analysis by comparing the expected and measured decrease due to the geomagnetic cutoff attenuation of charged particles. This correction is also included in this analysis by a shift of the energies in the CAL data.

To mitigate the inclusion of spurious events and overestimation of the exposure for the purposes of validation, we studied the distribution of photon candidates in the CAL coordinate frame in conjunction with the mapping of ISS structures and identified a region that is not significantly affected (Figure 10). The bottom half of the full FOV periodically views the solar panels, and the SSRMS remained for some time in the upper-left quadrant. However, in the shaded region (within the $45^{\circ} \mathrm{FOV}$ ) of Figure 10, the contamination is found to be negligible. To compare the distribution of expected events based on Fermi-LAT data with observations, the FOV of the CAL was limited to this region in the selection of event candidates and generation of the exposure. We note that the integrated effective area of the CAL is decreased by a factor of $\sim 4$ with this restriction and that the statistics for the following results are limited compared to an anticipated future analysis fully accounting for the obstructions.

The number of expected photons as a function of energy and sky position is calculated by applying the CAL exposure to the Fermi-LAT flux map. The observed gamma-ray candidates are tabulated and both data sets are restricted $\left(|\ell|<80^{\circ}\right)$ to remove

\footnotetext{
${ }^{50}$ https://fermi.gsfc.nasa.gov/ssc/data/access/lat/
} 


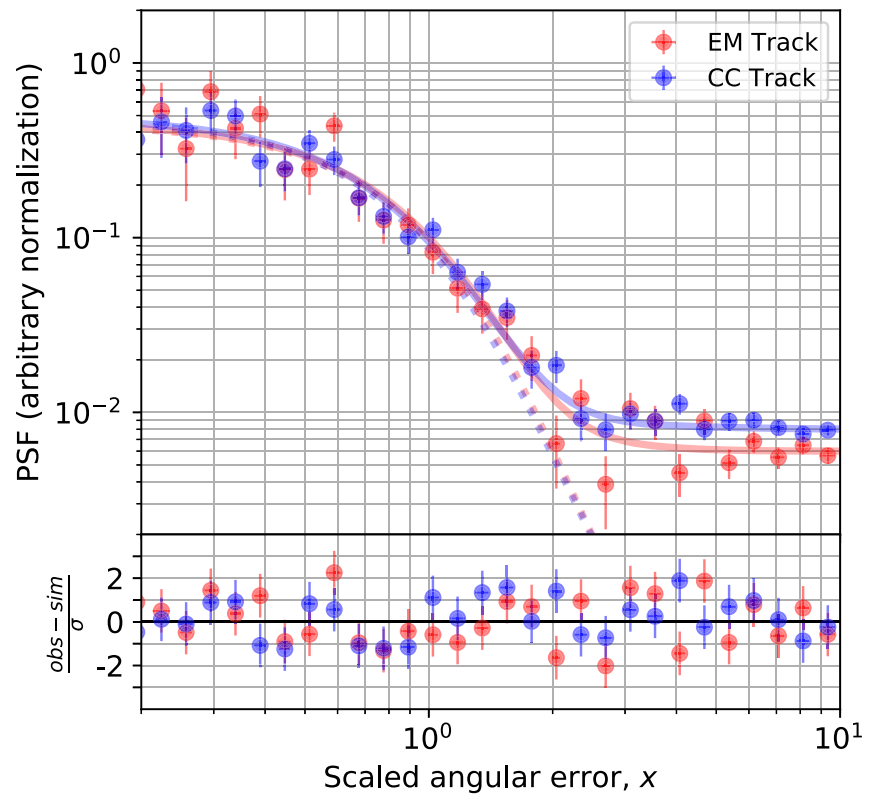

Figure 9. Flight data PSF distribution constructed using events near the Geminga source position. The effect of a constant background can be seen in the plateau for $x \gtrsim 2$ (dotted line shows the PSF with no background). In the lower plot, closed circles show the error in standard deviations after compensating for the background.

the contributions from the Crab, Geminga, and Vela pulsars. Projecting these results to galactic latitude, we obtain the distributions in Figure 11.

Comparing our observations to the expectation based on the LAT data, we find a chi-squared value of 88.34 (150.0) with 86 (89) degrees of freedom (d.o.f.) for the EM Track (CC Track). While there is no significant deviation from the expectation for the EM Track, the CC Track demonstrates a residual background at higher latitudes. We confirm this by recalculating the chi-squared for the CC Track restricted to $|b|<20^{\circ}$, obtaining a chi-squared of 19.15 with 20 d.o.f. Compared to previous presentations of CALET observations (Cannady et al. 2017; Mori et al. 2017) where a background component proportional to the exposure was subtracted, the masking of ISS structures is sufficient to achieve consistency for the EM Track distribution.

One significant contributor to the discrepancy in the $\mathrm{CC}$ Track result is the bright flare of CTA 102 (Figure 12) in 2016 November into early 2017. If we remove a window of $5^{\circ}$ around the CTA 102 position, the chi-squared values decrease to 77.96 and 133.6 for the EM Track and CC Track, respectively. The remaining background scales roughly with the exposure and is expected to comprise FOV obstructions that are not yet masked and residual charged particle contamination.

\subsection{Simulated Electron and Proton Contamination}

In order to assess the contamination from charged particles in the gamma-ray sample, electron and proton events simulated with EPICS/Cosmos are used to isolate a contaminating subset for the gamma-ray selection. The electron events are weighted to the CALET flux (Adriani et al. 2017), whereas the proton sample is weighted to PAMELA fluxes (Adriani et al. 2015) at energies below $30 \mathrm{GeV}$ and the larger of the AMS-02 (Aguilar et al. 2015) and CREAM-III (Yoon et al. 2017) parameterizations at higher energies. At low energies, the assumed electron flux is averaged based on the fraction of observation time spent in different L-shell regions. The PAMELA proton flux is similarly weighted, using bins in AACGM (altitude adjusted corrected geomagnetic) latitude rather than L-shell values. The kinetic energies of the protons and electrons are reconstructed as if they were gamma-ray primaries to match the energy scales for comparison.

To compare the simulated contaminating flux with the flux of the galactic and isotropic diffuse components, we segment the sky into regions considered on plane $\left(|\ell|<80^{\circ},|b|<8^{\circ}\right)$ and off plane $\left(|b|>10^{\circ}\right)$. Using HEALPix to segment the sky, the flux measured with the CAL is calculated in each pixel. Weighting by the exposure, the averages for the off- and onplane regions are determined as a function of energy. The simulated contamination is found to be on the order of $10 \%$ of the off-plane diffuse flux for energies up to $\sim 12 \mathrm{GeV}$ and negligible at higher energies.

For comparison, we average the Fermi-LAT fluxes using the same HEALPix scheme and exposure weighting as for the CAL data. We find the flux on plane to be consistent between the two data sets, although there is a noticeable excess in CAL data off plane at lower energies (Figure 13). The discrepancy and the simulated contaminating flux are similar in scale and shape, although an offset exists between fine features. In addition, at energies $<3 \mathrm{GeV}$, there remains an additional background component in the averaged fluxes in the CAL relative to FermiLAT. While we suspect that an unmodeled charged particle contribution is responsible for this difference, it is possible that unaccounted-for passages of ISS structures through the FOV occurred, and we are continuing to investigate the source.

The chi-squared statistics for the on-plane fluxes as compared to the LAT expectation are 16.5 with 19 d.o.f. and 5.31 with 10 d.o.f. for EM Track and CC Track, respectively. The agreement off plane is adversely affected by the residual background. This result does not yet include the removal of point sources and we anticipate a more robust result in the future with further background modeling and the opening of the full CAL FOV.

\subsection{Measurement of Bright Point Sources}

To further investigate the consistency of our observations with those by Fermi-LAT, we isolate events near the positions of the Crab, Vela, and Geminga pulsars. Figure 8 shows the events near the source position with the catalog and quaternioncorrected positions. Figure 12 shows maps of photon candidates seen by the CAL for EM Track and CC Track with the positions of the bright observed sources labeled. The spectra of the pulsars are calculated using events with scaled angular distance $x<2.6$ for EM Track and $x<3.4$ for CC Track (corresponding to $99 \%$ photon containment in the PSF) from the source positions. An energy-dependent background is removed from each source by scaling the number of events in an annulus with $4.5<x<6.5$ appropriately to match the size of the source window.

The resulting spectra (Figure 14) are tested for consistency with parameterized LAT spectra (Abdo et al. 2009a, 2009b, 2010). The difference between the parameterizations and the CALET fluxes are shown on the lower axes in units of the CAL 

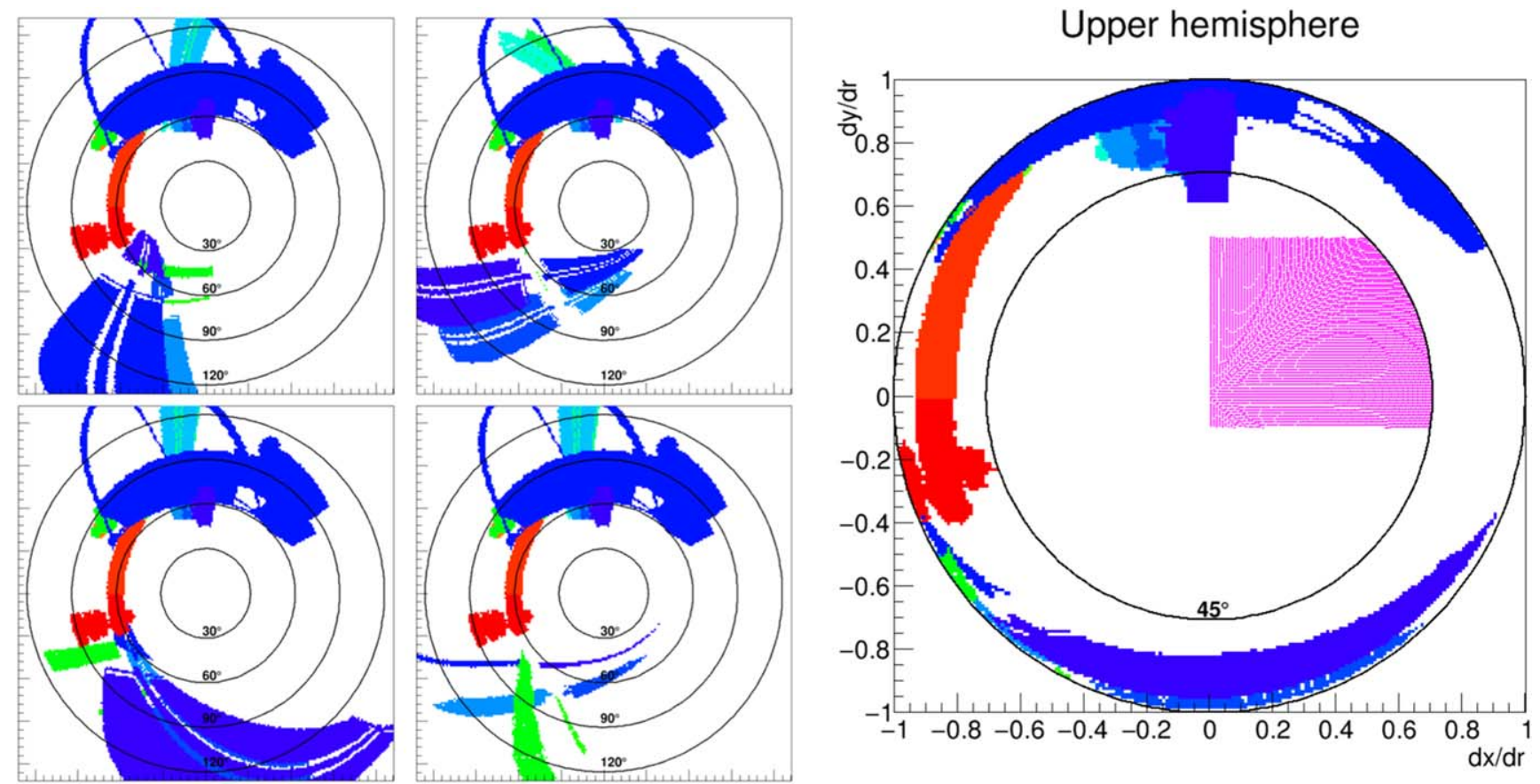

Figure 10. Left: frames showing the movement of the ISS solar panels and radiator in the CALET coordinate frame. Circles give the angle from the normal in intervals of $30^{\circ}$. Right: the CAL FOV for a sample orientation of the ISS structures. The approximately rectangular magenta shaded region is found to be mostly clear of obstructions for the first two years of observation data.

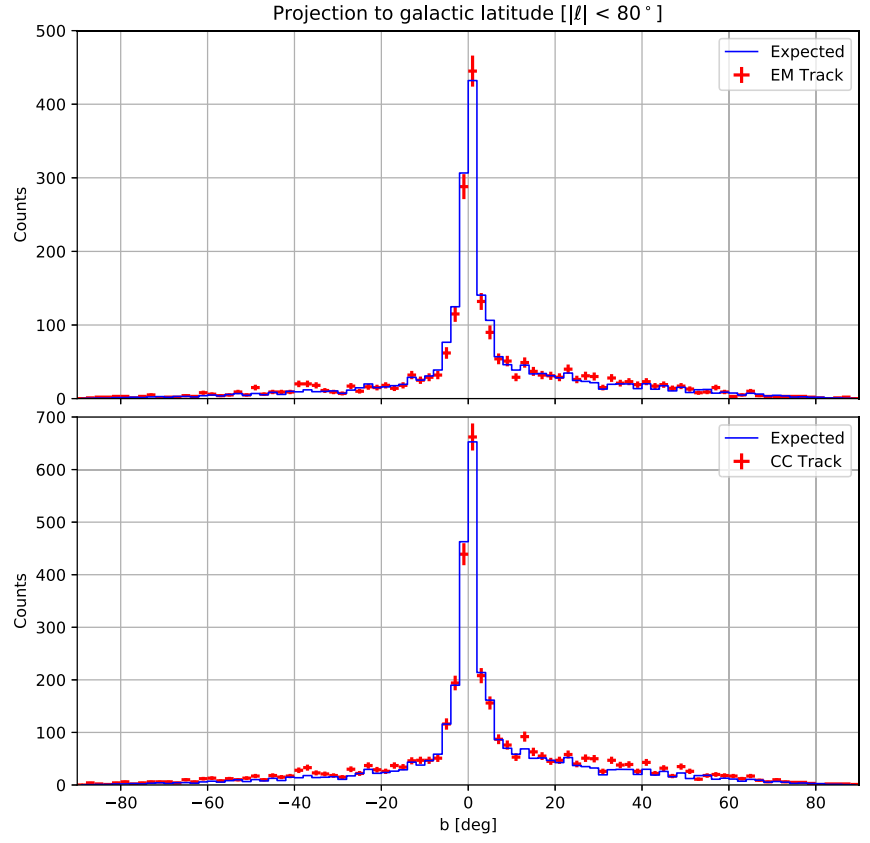

Figure 11. Projections of the observed and expected number of photons onto galactic latitude for the galactic plane region $|\ell|<80^{\circ}$ for EM Track and CC Track for the energy range $1 \mathrm{GeV}<100 \mathrm{GeV}$.

measurement error and data. Error bars shown are statistical only. Although Crab suffers from a relatively high background fraction, after subtraction, the derived flux is found to be consistent with the LAT spectrum, with chi-squared statistics 4.64 and 4.16, both with 7 d.o.f., for the EM Track and CC Track, respectively. Vela is observed consistently at the edge of the CAL FOV and may be subject to systematic effects in the exposure and in the determination of the background.
Geminga is observed with a very high signal over the background and is shown to be consistent with the Fermi-LAT spectrum with EM Track and CC Track chi-squared statistics of 6.73 and 5.74, respectively, with 8 d.o.f. We performed a chisquared fitting for the Geminga observations with a power-law, broken power-law, and cutoff power-law templates. We find that the observations are inconsistent with a pure power law and that the cutoff power law is slightly favored over a broken power law. The best fit obtained with the CALET-CAL data is a cutoff power law with spectral index $\alpha=1.19_{-0.51}^{+0.47}$ and cutoff energy $E_{c}=2.04_{-0.54}^{+0.92}$, consistent within errors with the FermiLAT fit.

\section{Search for Unknown Gamma-Ray Transients}

The highly successful multiwavelength campaign of observations of the neutron star-neutron star merger event GW 170817 and the associated electromagnetic detections clearly demonstrate the value of having multiple instruments capable of viewing different regions of the sky and different energy regimes simultaneously (Abbott et al. 2017a). The observation of the short gamma-ray burst (sGRB) event initially by Fermi GBM (von Kienlen et al. 2017) and subsequently by INTEGRAL (Abbott et al. 2017b) confirmed the association of at least some sGRBs with binary neutron star merger events and provided initial localization of the event, together with the LIGO-Virgo analysis, that guided the subsequent optical searches, especially the initial Swope telescope detection (Coulter et al. 2017). Given the redshift and compared to typical events, GRB 170817A was underluminous (and not detected at $\mathrm{GeV}$ energies), leading to questions about the universality of binary neutron star mergers as progenitors of sGRB events and the dependence of the high-energy emission on the existence of a jet and its viewing angle. Further observations at $\mathrm{GeV}$ energies of the prompt component of both sGRBs and long GRBs (whose emission mechanisms at high 

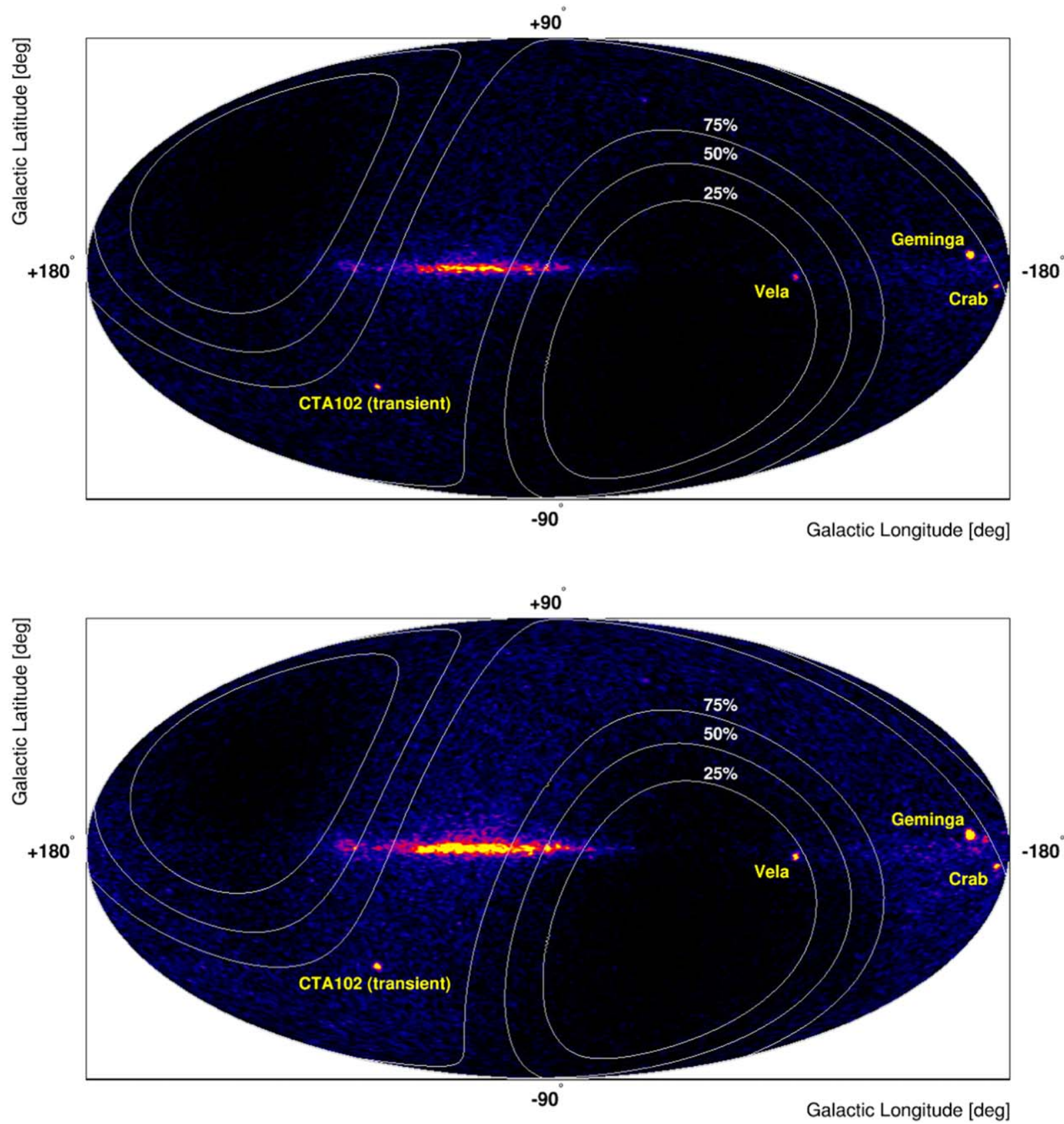

Figure 12. Signal maps for the EM Track (top) and CC Track (bottom) shown in a Mollweide projection of galactic coordinates. White contours show the relative level of exposure compared to the maximum on the sky. The Crab, Geminga, and Vela pulsars are clearly visible, as is a flare of the AGN CTA 102.

energies are thought to be similar despite different progenitor systems) will be critical in understanding these phenomena and can provide the localization required for multiwavelength follow-up studies.

In addition to the CGBM sensitivity to GRBs at $7 \mathrm{keV}-$ $20 \mathrm{MeV}$, the CAL provides sensitivity at $\mathrm{GeV}$ energies to transient events. To search for GeV-energy counterpart emission from such sources detected by other instruments, we check the CAL data at the reported trigger times for gamma-ray candidates. For events checked using CGBM, Swift, and Fermi/GBM triggers, and for the LIGO-Virgo gravitational wave $(\mathrm{GW})$ events, no significant counterparts have been detected at this stage for timescales ranging from $1 \mathrm{~s}$ to $1 \mathrm{hr}$. Additionally, we have developed an algorithm for the discovery of unlocalized transients using only the CAL data, which is described below.

\subsection{Methodology}

In order to search for and locate unknown gamma-ray transients with CALET, it is necessary to detect GeV-energy photons with the CAL since CGBM observations do not provide position information. In order to do this, we require two or more gamma-ray candidate events spatially coincident with the assumption of a common origin. Restricted time windows are imposed on the order of minutes to seconds, minimizing the background in the observations and increasing the likelihood that the events are associated. 

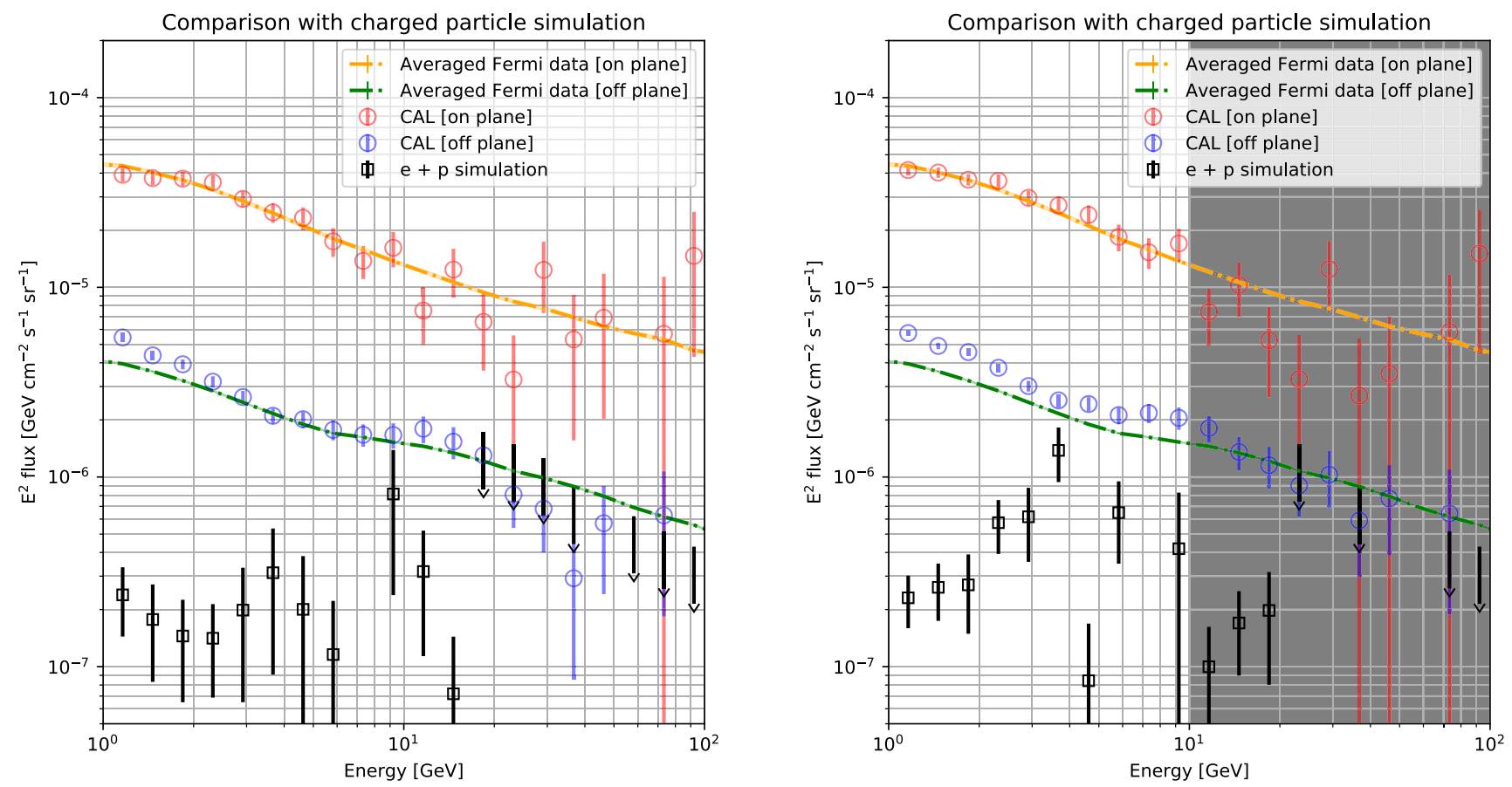

Figure 13. Fluxes obtained from exposure-weighted averages of the observations of the CAL on plane and off plane (left: EM Track, right: CC Track-valid up to $10 \mathrm{GeV}$ ). For comparison, the expectation based on Fermi-LAT data is shown by the green and orange curves. The estimated background using simulated charged particle (electron + proton) contamination is shown in black.

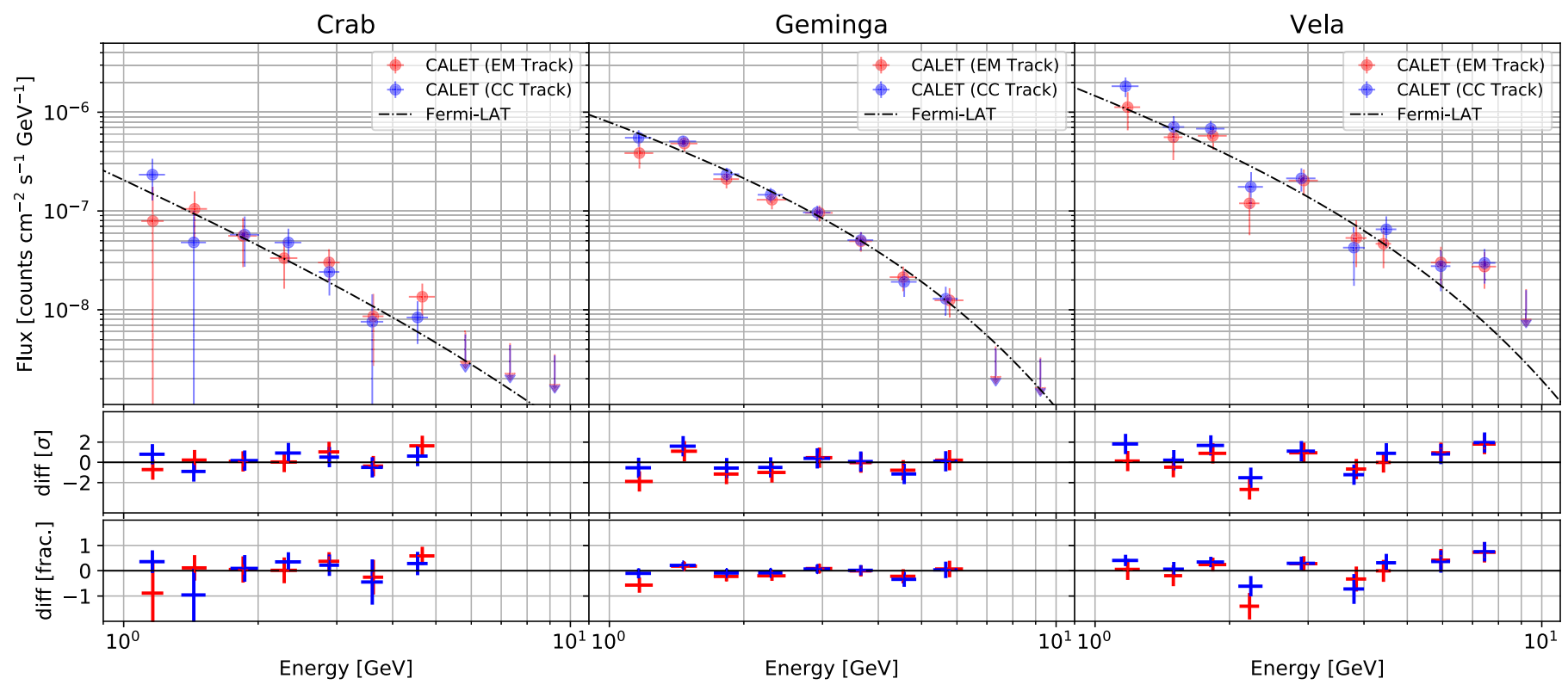

Figure 14. Source spectra for Crab, Geminga, and Vela. Background subtraction has been performed based on an energy-dependent estimation using events in an annulus around the source. Events were chosen using the scaling functions $S_{p}$ and the angular deviations such that the events associated within the $99 \%$ containment radius and background events have $4.5<x<6.5$. The lower panels in each plot show the difference between the CAL observations and the parameterization in units of CAL error (top) and fractional difference (bottom).

Search algorithm. To quantitatively estimate the probability of association for two events, we assume a common source and use the PSF derived in Section 4.1. The value of the scaling function $S_{p}$ is determined for each event based on its observed kinetic energy and the number of IMC layers used in its track reconstruction $\left(N_{p}\right)$. We generate a large sample set of simulated arrival directions by repeatedly (1) sampling a scaled error $x$ for each event using the PSF as the probability density, (2) scaling this back to a polar angle in degrees with the appropriate $S_{p}$, (3) randomly choosing an azimuthal angle from a uniform distribution on $[0,2 \pi)$ for each event, and (4) calculating the angular separation of the events based on the random positions.

By simulating many such sample pairs, we generate the probability distribution as a function of angular separation. If the opening angle between the two observed photons is less than the $68 \%$ containment radius according to the simulated probability distribution, the events are recognized as a pair.

False alarm rate. To determine the false alarm rate (FAR) for the method, we divide the flight data with the LE- $\gamma$ trigger 

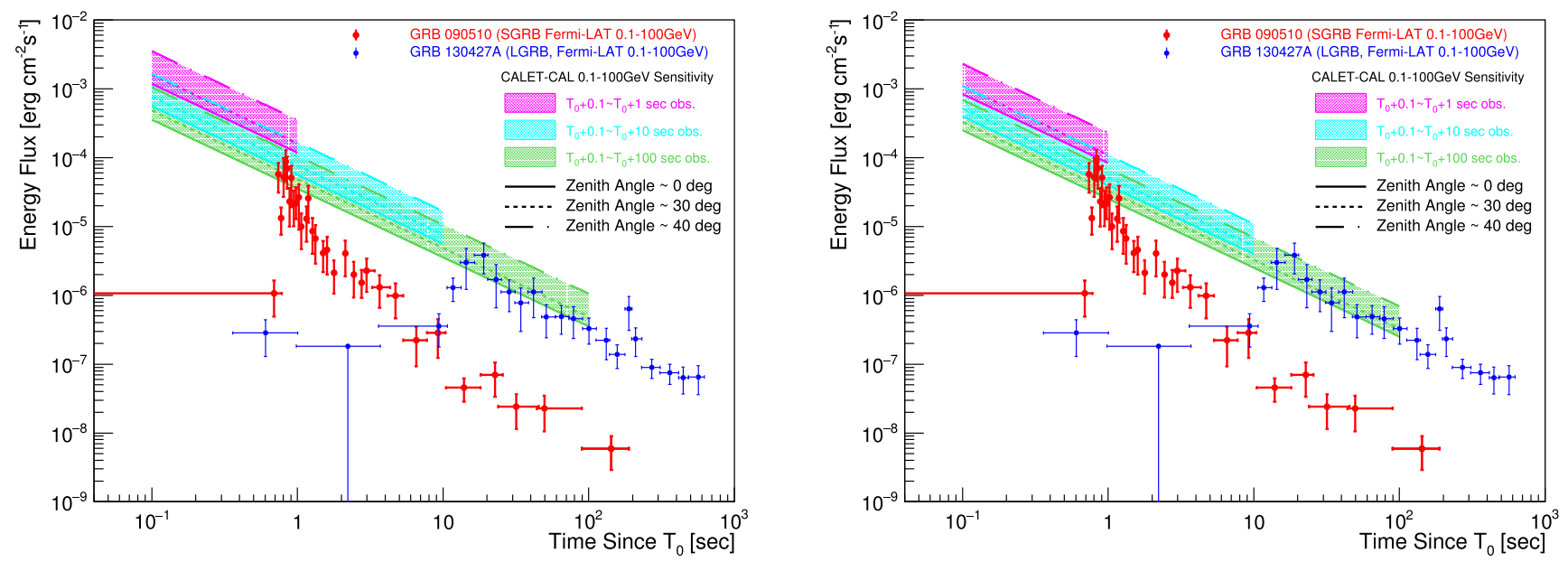

Figure 15. The expected sensitivity of the transient event search with the CAL for time windows of 1,10 , and $100 \mathrm{~s}$ at zenith angles of $0^{\circ}, 30^{\circ}$, and $40^{\circ}$. For comparison, the light curves for Fermi-LAT observations of the short GRB 090510 and the long GRB $130427 \mathrm{~A}$ are shown. The sensitivity calculation assumes an $E^{-2}$ spectrum and a $t^{-1}$ decay using the energy range $1-10 \mathrm{GeV}$. EM Track sensitivity is shown in the left frame and CC Track sensitivity in the right.

active into consecutive time windows and search for event pairs. With 100,884 trials of $100 \mathrm{~s}$ time windows, we find 25 pairs. This corresponds to an FAR of $0.025 \%$ for events with $E>1 \mathrm{GeV}$. In the case of 10,351 trials of 10 minute time windows, it increases to $0.48 \%$. Since LE- $\gamma$ flight data are used in this calculation, we note that real event pairs could be contained and identified in this evaluation and that the results should be taken as conservative estimates. In addition, the strict FOV mask described in Section 5.2 is not used in this calculation. With the implementation of robust ISS structure filtering in development, this FAR is expected to be further reduced.

\subsection{Automated Search}

In order to search for transients in a semi-real-time manner, an automatic transient search system is currently in development. Upon receipt of Level 0 scientific base data, the processing to Level 1 analysis data described in Asaoka et al. (2018) is automatically triggered. A search for gamma rays is performed, and the resulting candidates are then fed through the search as described above. If event pairs are identified, then further analysis can be performed.

Since production of Level 2 data is very CPU intensive (requiring $10 \mathrm{hr}$ to process $1 \mathrm{hr}$ of flight data on a single CPU), it is necessary to parallelize this task for the purpose of the automated search. A prototype system is running at the Waseda CALET Operations Center. It is currently in the validation stage for simulated GRB injections and for long-term stability. Once the system is successfully established, our ultimate goal is to provide transient alerts to the gamma-ray community.

\subsection{Expected Sensitivity}

Figure 15 shows the expected sensitivity for the discovery of unknown $\mathrm{GeV}$ gamma-ray transients with the CAL. The assumed spectrum for emission in the figure is $E^{-2}$ starting at $T-T_{0}=0.1 \mathrm{~s}$, and the light curve is assumed to decrease inversely with time. The sensitivity is shown for time windows of 1,10 , and $100 \mathrm{~s}$ with source zenith angles of $0^{\circ}, 30^{\circ}$, and $40^{\circ}$ in the CAL FOV. The light curves for the Fermi-LAT detections of GRB 090510 (Ajello et al. 2018) and GRB 130427A (Ackermann et al. 2013) for the energy range
$0.1-100 \mathrm{GeV}$ are shown for comparison. Despite the lack of sensitivity to sub-GeV photons in the CAL, the $0.1-1 \mathrm{GeV}$ band is included in this calculation of the limit to compare to the Fermi-LAT light curve since the energy flux is sensitive to the range over which it is integrated. The limit for the $1-10 \mathrm{GeV}$-energy band only in the CAL is lower than that shown in the figure by roughly a factor of 3 .

When CGBM triggers on a GRB event, the signal is sent to the CAL, and the CAL trigger threshold is reduced to the $\sim 1 \mathrm{GeV}$ level. This enables the CAL to search for counterpart $\mathrm{GeV}$ emission from CGBM-triggering GRBs even if the LE- $\gamma$ would typically not be active.

To detect the prompt emission from a short GRB event similar to GRB 090510, the source would need to be observed near the zenith in the CAL FOV. Given that the spectrum for this GRB is harder than the assumed $E^{-2}$ power law, it is possible that the prompt emission could be detected if the source were near the center of the CAL FOV at the trigger time. Given the longer timescale of high-energy emission for long GRBs, the potential for discovery for the CAL is thus higher. For an event with energy flux similar to GRB 130427A, we could expect to localize the source with up to $\sim 3$ photons for $138 \mathrm{~s}$ of observation assuming the time-resolved spectra in Tam et al. (2013), depending on the location of the event in the FOV and its path over the observation time.

With regard to searching for electromagnetic counterparts to LIGO/Virgo gravitational wave events, we note that GRB 090510 is reported to have redshift $z=0.9$, whereas the current $\mathrm{GW}$ triggers are mostly within $z=0.1$. For an event with luminosity similar to that of GRB 090510 occurring at a distance $z \sim 0.1$ in the CAL FOV, we anticipate that a significant signal would be detected.

\section{Conclusion}

The instrument response of the CALET calorimeter to gamma rays has been studied using both simulated and on-orbit data for the first two years of scientific operations (for LE- $\gamma$, 2015 November through 2017 October; LE- $\gamma$ mode was not active for the month 2015/10, although CALET was observing). We calculate the effective area and angular resolution of the CAL and derive the PSF from simulated data. The 
simulated result is found to be consistent with the measured signal from bright point sources in the flight data.

Consistency of the distribution of events from a limited FOV window as a function of galactic latitude with the expectation from Fermi-LAT data using the EM Track algorithm is demonstrated, while a residual unresolved background is present in the off-plane regions for the CC Track. In addition, we find the fluxes averaged in the galactic plane region with this same restricted data set to be consistent with Fermi-LAT data. The residual background with the strict FOV mask is demonstrated to be on the same order as the simulated contamination from charged particles, but the inherent variation in the energy deposited in the calorimeter by hadronic showers and the sensitivity to the input particle spectrum preclude a clear association. Extension of these measurements to the full FOV of the CAL is in progress, pending complete characterization of moving ISS structures, which regularly introduce a significant photon background into the instrument.

Measurement of the energy spectra for the Crab, Geminga, and Vela pulsars using the full FOV with an energy-dependent background component subtracted demonstrates the sensitivity of the calorimeter to observe bright, persistent sources. Furthermore, the signal from these sources was used to validate the PSF as derived from simulated data with on-orbit observations. With increased exposure over the planned fiveyear lifetime of CALET and the lower background due to upcoming improvements in the ISS structure veto, the spectra of these bright pulsars will be extended beyond $10 \mathrm{GeV}$ and an independent search for dimmer gamma-ray sources is possible.

These results establish the capability of the CALET calorimeter to observe gamma rays in the energy range $\sim 1 \mathrm{GeV}$ to over $100 \mathrm{GeV}$. Further refinement of the galactic and source measurements is anticipated with the veto of the FOV obstructions. Characterization of the HE trigger is underway and will extend the CAL observation period to times when the LE- $\gamma$ trigger is not active. With the instrument response functions characterized, we also anticipate the detailed study of individual sources and, upon deployment of the near-real-time transient search, hope to provide alerts to the gamma-ray community in the near future.

We gratefully acknowledge JAXA's contributions to the development of CALET and to the operations on board the ISS. We also wish to express our sincere gratitude to ASI and NASA for their support of the CALET project. This work is partially supported by JSPS Grant-in-Aid for Scientific Research (S) Number 26220708, JSPS Grant-in-Aid for Scientific Research (B) Number 17H02901, JSPS Grant-in-Aid for Scientific Research (C) Number 16K05382 and MEXT-Supported Program for the Strategic Research Foundation at Private Universities (2011-2015) S1101021 in Waseda University. This work is also supported in part by MEXT Grant-in-Aid for Scientific Research on Innovative Areas Number 24103002. US CALET work is supported by NASA under RTOP 14-APRA14-0075 (GSFC) and grants NNX16AC02G (WUSL), NNX16AB99G (LSU), and NNX11AE06G (Denver). High-statistics EPICS/Cosmos data were generated on the SuperMike-II cluster maintained at the High Performance Computing facilities at Louisiana State University.
Software: astropy (Robitaille et al. 2013), numpy (van der Walt et al. 2011), matplotlib (Hunter 2007), HEALPix (Gorski et al. 2005), CERN ROOT (Brun \& Rademakers 1997).

\section{ORCID iDs}

N. Cannady (ib https://orcid.org/0000-0003-2916-6955

Y. Asaoka (iD https://orcid.org/0000-0001-6440-933X

S. Torii (iD https://orcid.org/0000-0003-3363-3827

M. L. Cherry (iD https://orcid.org/0000-0003-2808-312X

M. Mori (iD https://orcid.org/0000-0003-2921-1592

O. Adriani (1) https://orcid.org/0000-0002-3592-0654

K. Asano (1) https://orcid.org/0000-0001-9064-160X

W. R. Binns (i) https://orcid.org/0000-0001-6110-3407

M. Bongi (iD https://orcid.org/0000-0002-6050-1937

V. Di Felice (iD https://orcid.org/0000-0002-6404-6177

K. Ebisawa (iD https://orcid.org/0000-0002-5352-7178

T. G. Guzik (iD https://orcid.org/0000-0001-6339-8261

K. Ioka (i) https://orcid.org/0000-0002-3517-1956

M. H. Israel (iD https://orcid.org/0000-0002-8104-208X

K. Kasahara (iD https://orcid.org/0000-0001-5611-3301

J. Kataoka (i) https://orcid.org/0000-0003-2819-6415

R. Kataoka (ib https://orcid.org/0000-0001-9400-1765

N. Kawanaka (iD https://orcid.org/0000-0001-8181-7511

H. S. Krawczynski (iD https://orcid.org/0000-0002-1084-6507

S. Miyake (iD https://orcid.org/0000-0002-3067-655X

N. Mori (i) https://orcid.org/0000-0003-2138-3787

K. Munakata (iD https://orcid.org/0000-0002-2131-4100

L. Pacini (ib https://orcid.org/0000-0001-6808-9396

B. F. Rauch (1) https://orcid.org/0000-0002-1452-4142

S. B. Ricciarini (10 https://orcid.org/0000-0001-6176-3368

R. Sparvoli (i) https://orcid.org/0000-0002-6314-6117

J. E. Suh (ib https://orcid.org/0000-0003-1024-2292

Y. Tsunesada (i) https://orcid.org/0000-0001-9238-6817

S. Yanagita (i) https://orcid.org/0000-0002-5837-8511

\section{References}

Abbott, B. P., Abbott, R., Abbott, T. D., et al. 2017a, ApJL, 848, 12 Abbott, B. P., Abbott, R., Abbott, T. D., et al. 2017b, ApJL, 848, 13 Abdo, A. A., Ackermann, M., Ajello, M., et al. 2009b, ApJ, 708, 1254 Abdo, A. A., Ackermann, M., Ajello, M., et al. 2010, ApJ, 720, 272 Abdo, A. A., Ackermann, M., Atwood, W. B., et al. 2009a, ApJ, 696, 1084 Acero, F., Ackermann, M., Ajello, M., et al. 2015, ApJS, 218, 23 Ackermann, M., Ajello, M., Albert, A., et al. 2012, ApJS, 203, 4 Ackermann, M., Ajello, M., Asano, K., et al. 2013, Sci, 343, 42 Adriani, O., Akaike, Y., Asano, K., et al. 2016, ApJL, 829, 1 Adriani, O., Akaike, Y., Asano, K., et al. 2017, PhRvL, 119, 181101 Adriani, O., Barbarino, G. C., Bazilevskaya, G. A., et al. 2015, JGR, 120, 3728 Aguilar, M., Aisa, D., Alpat, B., et al. 2015, PhRvL, 114, 171103 Ajello, M., Allafort, A., Axelsson, M., et al. 2018, ApJ, 861, 85

Akaike, Y. for the CALET Collaboration 2013, Proc. ICRC, 0726, 2162 Akaike, Y. for the CALET Collaboration 2015, Proc. ICRC (The Hague), 34, 613 Asaoka, Y., Akaike, Y., Komiya, Y., et al. 2017, APh, 91, 1 Asaoka, Y., Ozawa, S., Torii, S., et al. 2018, APh, 100, 29

Brun, R., \& Rademakers, F. 1997, NIMPA, 389, 81

Cannady, N. for the CALET Collaboration 2017, Proc. ICRC (Busan), 35, 720 Coulter, D. A., Kilpatrick, C. D., Siebert, M. R., et al. 2017, GCN, 21529

Górski, K. M., Hivon, E., Banday, A. J., et al. 2005, ApJ, 622, 759

Hunter, J. D. 2007, CSE, 9, 90

Jørgensen, J. L., \& Liebe, C. C. 1996, AcAau, 39, 775

Kasahara, K. 1995, Proc. ICRC (Rome), 1, 399

King, I. 1962, AJ, 67, 471

Maestro, P., \& Mori, N. for the CALET Collaboration 2017, Proc. ICRC (Busan), 35, 208 
Mori, M., \& Asaoka, Y. for the CALET Collaboration 2017, Proc. ICRC (Busan), 35, 637

Mori, M. for the CALET Collaboration 2013, Proc. ICRC (Rio de Janeiro), 0248,1185

Robitaille, T. P., Tollerud, E. J., Greenfield, P., et al. 2013, A\&A, 558, A33

Sullivan, J. D. 1971, NucIM, 95, 5

Tam, P. T., Tang, Q., Hou, S., Liu, R., \& Wang, X. 2013, ApJL, 771, 13
Torii, S. for the CALET Collaboration 2015, Proc. ICRC (The Hague), 34, 581

van der Walt, S., Colbert, S. C., \& Varoquaux, G. 2011, CSE, 13, 22

von Kienlen, A., Meegan, C., Goldstein, A. for the Fermi GBM Team 2017, GCN, 21520

Yamaoka, Kfor the CALET Collaboration 2017, Proc. ICRC (Busan), 35, 239

Yoon, Y. S., Anderson, T., Barrau, A., et al. 2017, ApJ, 839, 5 